\title{
Newton Binomial Formulas in Schubert Calculus
}

\author{
Jorge CORDOVEZ, Letterio GATTO, \\ and Taíse SANTIAGO
}

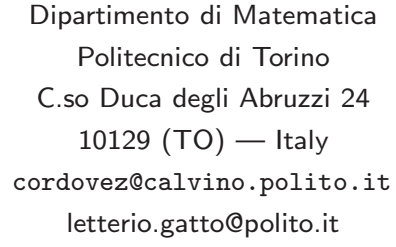

\author{
Instituto de Matemática \\ Universidade Federal da Bahia \\ Av. Ademar de Barros s/n, Ondina \\ Salvador (Bahia) 40170-110 (BA) - Brazil \\ taisesantiago@ufba.br
}

Received: November 15, 2007

Accepted: June 2, 2008

To Israel Vainsencher on the occasion of his 60th birthday

\begin{abstract}
We prove Newton's binomial formulas for Schubert Calculus to determine numbers of base point free linear series on the projective line with prescribed ramification divisor supported at given distinct points.
\end{abstract}

Key words: Schubert Calculus on a Grassmann algebra, Newton's binomial formulas in Schubert calculus, enumerative geometry of linear series on the projective line.

2000 Mathematics Subject Classification: 14M15, 14N15, 15A75.

\section{Introduction}

Let $G(k, n)$ be the complex Grassmannian variety parameterizing $k$-dimensional subspaces of $\mathbb{C}^{n}$. In [6] (see also $[7,17]$ ), the intersection theory on $G(k, n)$ (Schubert calculus) is rephrased via a natural derivation on the exterior algebra of a free $\mathbb{Z}$-module of rank $n$. Classical Pieri's and Giambelli's formulas are recovered, respectively, from Leibniz's rule and integration by parts inherited from such a derivation. The generalization of [6] to the intersection theory on Grassmann bundles is achieved in [8],

Work partially sponsored by PRIN "Geometria sulle Varietà Algebriche" (Coordinatore A. Verra), INDAM-GNSAGA, Politecnico di Torino, FAPESB (proc. n 8057/2006), CNPq (proc. n 350259/2006-2), UEFS - Brazil. 
by suitably translating previous important work by Laksov and Thorup [13,14] regarding the existence of a canonical symmetric structure on the exterior algebra of a polynomial ring.

It is natural to ask if the aforementioned derivation formalism for Schubert calculus is worthy or if it is nothing more than a mere translation of an old theory into a more or less new language. Indeed, a couple of years ago, K. Ranestad asked us to test our methods to compute (and possibly to find a formula for) the total number, with multiplicities, of non projectively equivalent rational space curves of degree $n+3$ having flexes at given $2 n$ distinct points. Any such a curve is the image of the morphism $\phi: \mathbb{P}^{1} \rightarrow \mathbb{P}^{3}$ induced by a very ample $g_{n+3}^{3}$ on $\mathbb{P}^{1}$, such that the ramification sequence (in the sense of $[12$, section 1.2$]$ ) at each of its ramification point is $(1,2,4,5)$. Results by Eisenbud and Harris [4] ensure that such a number is finite and equal to the degree of a suitable product of Schubert cycles (see section 4.9 for details). To compute it, we rely on the two main results of this paper, Theorem 2.5 and Theorem 2.6, regarding certain Newton's binomial formulas, which cannot be expressed within the classical Schubert calculus formalism (as, e.g., in [10]). Such formulas allow us to reduce, after finitely many steps, the degree of any top codimensional product of Schubert cycles into an explicit integral linear combination of degrees of Schubert varieties (the latter already computed by Schubert in [20]).

The referee, however, suggested us that the same invoked results of [4] lead naturally to generalize Ranestad's question (and its answer) to that of finding the number, with multiplicities, of $g_{d}^{r} s$ on $\mathbb{P}^{1}$ having pre-assigned ramification at prescribed distinct points. This last question was also raised by Osserman in [16], who conjectures that the scheme of such linear series is reduced when the points are chosen in general position. In the case $r=1$, I. Scherbak proves, via arguments borrowed from representation theory, a nice formula counting pencils on $\mathbb{P}^{1}$ with prescribed ramifications [18] (see also [19]), while in [16] some recursive formulas regarding the case of higher dimensional linear series are proven. Similar counting questions have also been studied by Kharlamov and Sottile in [12], in the more delicate context of real enumerative algebraic geometry.

Our paper is organized as follows. Section 2 states, proves, and discusses Newton formulas in Schubert calculus. They remarkably imply that to determine the product structure of $A^{*}(G(k, n))$ is sufficient to know only one Pieri's formula, namely that expressing the product of a generator of $A^{1}(G(k, n))$ with any Schubert cycle (see remark in section 2.7). In fact, the product of any special Schubert cycle (see [10]) with an arbitrary one, can be reduced to that primitive case. We do think that this observation is rather new.

In section 3, Newton's type formulas are then applied to compute certain powers of special Schubert cycles capped with arbitrary classes (modulo rational equivalence) of Schubert varieties. Such entirely formal and straightforward computations will finally be exploited in section 4 to count base point free $g_{d}^{r} \mathrm{~s}$ on $\mathbb{P}^{1}$, with certain prescribed ramification divisors, for $1 \leq r \leq 3$. In particular, we use the inclusion- 
exclusion formula (as done in [19] in the context of representation theory) to supply an alternative proof of (a slightly modified version of) Scherbak's formula within our framework (Theorem 4.5). The method we use seems to be easily generalizable to the case of Grassmannians of higher dimensional subspaces. However, we shall not follow that path, as we think it is easier to express an integral in $A_{*}(G(k, n))$ as a linear combination of degrees of Schubert varieties.

Combining the theory exposed in [4], or [12, section 1], with our Newton's formulas (Theorems 2.5 and 2.6), we are finally able to give explicit expressions for the total number $N_{a, b, c, d}$, with multiplicities, of plane irreducible rational curves of degree $n+2$ having a flexes, $b$ hyperflexes, c cusps, and $d$ tacnodes at $a+b+c+d$ distinct points, such that $a+2 b+2 c+3 d=3 n$. Similarly, we offer an expression for the number $f_{a, b, c, d}$ of space rational curves having a stalls, b hyperstalls (see 4.9), c flexes, and $d$ cusps at $a+b+c+d$ distinct points such that $a+2 b+2 c+3 d=4 n$. For instance, putting $a=b=d=0$, one gets

$$
f_{0,0,2 n, 0}=\sum_{\substack{c_{1}+c_{2}+c_{3}+c_{4}=2 n \\
0 \leq m \leq c_{1}+c_{2}}} \frac{(2 n) !}{c_{1} ! c_{2} ! c_{3} ! c_{4} !}\left(\begin{array}{c}
c_{1}+c_{2} \\
m
\end{array}\right) \omega_{I\left(c_{1}, \ldots, c_{4} ; m\right)},
$$

where $\omega_{I\left(c_{1}, \ldots, c_{4} ; m\right)}$ is, up to a sign, the degree of a certain Schubert variety explicitly described in section 4.11. Formula (1) is our answer to the original Ranestad's question. In the same vein, putting $a=c=d=0$, one obtains

$$
\mathrm{HS}_{n}=f_{0,2 n, 0,0}=\sum_{\substack{b_{1}+\cdots+b_{5}=2 n \\
0 \leq l \leq b_{2}+b_{3}}} \frac{(2 n) !}{b_{1} ! \cdots b_{5} !}\left(\begin{array}{c}
b_{2}+b_{3} \\
l
\end{array}\right) \omega_{I\left(b_{1}, \ldots, b_{5} ; l\right)},
$$

which is the formula expressing the number (with multiplicities) of (non projectively equivalent) space rational curves having hyperstalls at prescribed $2 n$ distinct points. Indeed, the r.h.s. of (2) is equal to the degree of the 0-dimensional cycle $\sigma_{2}^{2 n} \cap[G(4, n+4)]$, where $\sigma_{2}$ is the second Chern class of the universal quotient bundle sitting over $G(4, n+4)$. One may wish to compute the list of $\mathrm{HS}_{n}, n \geq 0$, by directly evaluating the above degree via a mere iteration of Pieri's formula (as, e.g., in [10]). However, computations get very messy already for small values of $n$. Moreover "Schubert," the celebrated Maple ${ }^{\circledR}$ package designed by Katz and Strømme [11], is apparently unable to go beyond $n=12$ (see [15]). Using Schubert2 on Macaulay2, by Grayson and Stillman [9], one can do much better: on a computer with cpu speed 2.2 Ghz, 16Gb RAM, 4Gb swap, Jan-Magnus Økland obtained the list of $\mathrm{HS}_{n}$ up to $n=40$, with $\mathrm{HS}_{40}$ running for about 8 hours. Our formula (2), by contrast, requires no special computer package to be evaluated, since it is nothing else than a sum of products of multinomials. In fact, we have been able to write a trivial CoCoA code, successively improved by Økland [3], to write a list of $\mathrm{HS}_{n}$ via (2). It computes $\mathrm{HS}_{42}$ to be

201517182255943002813954873119143476157329393137457696988123090973997900 
in about half a hour, showing that the high computational efficiency of our formulas is out of reach by the up to now available packages for doing Schubert calculus.

\section{Preliminaries}

1.1. Let $X$ be an indeterminate over $\mathbb{Z}, M_{n}:=X \mathbb{Z}[X] /\left(X^{n+1}\right)$ and $\epsilon^{i}:=X^{i}+\left(X^{n+1}\right)$. As $m>n$ implies $\epsilon^{m}=0_{M_{n}}$, the $\mathbb{Z}$-module $M_{n}$ is freely generated by $\epsilon^{n}=\left(\epsilon^{1}, \ldots, \epsilon^{n}\right)$. Let $\mathbf{n}$ be the set of the first $n$ positive integers. The weight of $I:=\left(i_{1}, \ldots, i_{k}\right) \in \mathbf{n}^{k}$ is $\operatorname{wt}(I):=\sum_{j=1}^{k}\left(i_{j}-j\right)=\sum_{j=1}^{k} i_{j}-\frac{1}{2} k(k+1)$ (a non negative integer if the entries of $I$ are all distinct). In $\bigwedge^{k} M_{n}$, the $k^{\text {th }}$ exterior power of $M_{n}$, we shall often write $\boldsymbol{\epsilon}^{I}$ instead of the longer expression $\epsilon^{i_{1}} \wedge \cdots \wedge \epsilon^{i_{k}}$. Let $I^{\tau}:=\left(i_{\tau(1)}, \ldots, i_{\tau(k)}\right)$, where $\tau \in S_{k}$, the symmetric group on $k$ elements: then $\boldsymbol{\epsilon}^{I}=\boldsymbol{\epsilon}^{I^{\tau}}$. In particular, $\boldsymbol{\epsilon}^{I}=0$ if at least two entries of $I$ are equal. If $\boldsymbol{\epsilon}^{I} \neq 0$, the equality $\operatorname{wt}\left(\boldsymbol{\epsilon}^{I}\right):=\operatorname{wt}(I)$ defines the weight of $\epsilon^{I} \in \bigwedge^{k} M_{n}$.

1.2. Let $\mathcal{I}_{n}^{k}=\left\{I:=\left(i_{1}, \ldots, i_{k}\right) \in \mathbf{n}^{k} \mid 1 \leq i_{1}<\cdots<i_{k} \leq n\right\}$ be the lexicographically (totally) ordered set of all the strictly increasing sequences of $k$ positive integers not bigger than $n$. We also write $\mathcal{I}_{n}^{k, w}$ for the set of all $I \in \mathcal{I}_{n}^{k}$ such that $\operatorname{wt}(I)=w$. Let $\Lambda^{k} \epsilon^{n}:=\left(\epsilon^{I}: I \in \mathcal{I}_{n}^{k}\right)$ be the natural $\mathbb{Z}$-basis of $\Lambda^{k} M_{n}$ induced by $\boldsymbol{\epsilon}^{n}$. Denote by $\left(\bigwedge^{k} M_{n}\right)_{w}$ the free submodule of $\bigwedge^{k} M_{n}$ generated by the elements of $\bigwedge^{k} \epsilon^{n}$ of weight $w$. Clearly $\left(\bigwedge^{k} M_{n}\right)_{w}=0$ if $w>k(n-k)$. Then $\bigwedge^{k} M_{n}$ gets a structure of graded $\mathbb{Z}$-module, $\bigwedge^{k} M_{n}=\bigoplus_{w \geq 0}\left(\bigwedge^{k} M_{n}\right)_{w}$.

1.3. The fundamental element of $\Lambda^{k} M_{n}$ is the unique element $g_{k, n} \in \Lambda^{k} \epsilon^{n}$ of weight 0 , i.e., $g_{k, n}:=\epsilon^{1} \wedge \cdots \wedge \epsilon^{k}$. The point element of $\wedge^{k} M_{n}$ is the unique element $\pi_{k, n} \in \bigwedge^{k} \epsilon^{n}$ of weight $k(n-k)$, i.e., $\pi_{k, n}:=\epsilon^{n-k+1} \wedge \cdots \wedge \epsilon^{n}$.

1.4. By [8], there is a unique sequence $D:=\left(D_{0}, D_{1}, \ldots\right)$ of endomorphisms of $\wedge M_{n}$, the exterior algebra of $M_{n}$, such that, for each $\mathbf{p}, \mathbf{q} \in \Lambda M_{n}$, each $h, l \geq 0$, and each $i \geq 1$,

$$
\left\{\begin{array}{cr}
D_{h}(\mathbf{p} \wedge \mathbf{q})=\sum_{\left\{h_{1}, h_{2} \geq 0 \mid h_{1}+h_{2}=h\right\}} D_{h_{1}} \mathbf{p} \wedge D_{h_{2}} \mathbf{q} & \left(h^{\text {th }} \text { Leibniz's rule) },\right. \\
D_{l} \epsilon^{i}=\epsilon^{i+l} & \text { (initial conditions on } \left.\bigwedge^{1} M_{n}:=M_{n}\right) .
\end{array}\right.
$$

Equations (3) imply that $D_{h}\left(\bigwedge^{k} M_{n}\right)_{w} \subseteq\left(\bigwedge^{k} M_{n}\right)_{w+h}$. Thus $D_{h}$ is a homogeneous endomorphism of degree 0 of the exterior algebra. The induced endomorphism of $\bigwedge^{k} M_{n}$, for each $k \geq 1$, is homogeneous of degree $h$, with respect to the weight graduation of $\wedge^{k} M_{n}$. The initial conditions (3) and an easy induction show that the endomorphisms $\left\{D_{i}\right\}_{i \geq 0}$ are pairwise commuting: $D_{i} \circ D_{j}=D_{j} \circ D_{i}$ in $\bigwedge M_{n}$. 
1.5. For each $I \in \mathbf{n}^{k}$, the degree of $\boldsymbol{\epsilon}^{I}$,

$$
\int_{n} \boldsymbol{\epsilon}^{I}=\int_{n} \epsilon^{i_{1}} \wedge \cdots \wedge \epsilon^{i_{k}},
$$

is defined to be 1 (resp. - 1 ) if there is an even (resp. odd) permutation $\tau \in S_{k}$ such that $\epsilon^{I^{\tau}}=\pi_{k, n}$, and zero otherwise. In particular $\int_{n} \epsilon^{i_{1}} \wedge \cdots \wedge \epsilon^{i_{k}} \neq 0$ implies $\operatorname{wt}(I)=k(n-k)$. By linear extension one gets a function degree $\int_{n}: \bigwedge^{k} M_{n} \rightarrow \mathbb{Z}$, whose kernel is precisely the submodule of $\bigwedge^{k} M_{n}$ of elements of weight smaller than $k(n-k)$.

1.6. Let $\mathbb{Z}[\boldsymbol{T}]:=\mathbb{Z}\left[T_{1}, T_{2}, \ldots\right]$ be the polynomial ring in infinitely many indeterminates. Then $\mathbb{Z}[\boldsymbol{T}]=\bigoplus_{h \geq 0} \mathbb{Z}[\boldsymbol{T}]_{h}$, where one agrees that the degree of a monomial $a T_{i_{1}}^{j_{1}} \cdots T_{i_{h}}^{j_{h}}(a \in \mathbb{Z})$ is $i_{1} j_{1}+\cdots+i_{h} j_{h}$. If $I \in \mathcal{I}_{n}^{k}, \Delta_{I}(\boldsymbol{T}):=\operatorname{det}\left(T_{j_{i}-i}\right) \in \mathbb{Z}[\boldsymbol{T}]$ is the $(I, \boldsymbol{T})$-Schur determinant. It is clearly homogeneous of degree $\mathrm{wt}(I)$.

1.7. The Chow group $A_{*}(G(k, n))$ of the Grassmannian variety parameterizing $k$-planes in $\mathbb{C}^{n}$ is a free $\mathbb{Z}$-module of rank $\left(\begin{array}{l}n \\ k\end{array}\right)$ generated by $\left\{\Omega_{I} \mid I \in \mathcal{I}_{n}^{k}\right\}$, the cycle classes modulo rational equivalence of Schubert varieties $\Omega_{I}\left(E^{\bullet}\right)$ associated to some complete flag $E^{\bullet}$ of $\mathbb{C}^{n}$. The Chow ring $A^{*}(G(k, n))$ is a $\mathbb{Z}$-algebra generated by $\sigma:=\left(\sigma_{i}\right)_{i \geq 0}$, where $\sigma_{i}=c_{i}\left(\mathcal{Q}_{k}\right)$, the $i^{\text {th }}$ Chern class of the (rank $\left.n-k\right)$ universal quotient bundle on $G(k, n)$ (clearly $\sigma_{m}=0$ if $\left.m>n-k\right)$. Moreover $A_{*}(G(k, n)$ ) is a free module of rank 1 over $A^{*}(G(k, n))$ via cap product (Poincaré duality). The classical Giambelli's formula can be phrased by saying that $\Omega_{I}:=\Delta_{I}(\sigma) \cap[G(k, n)]$. In particular one sees that $A^{*}(G(k, n))$ is a free $\mathbb{Z}$-module generated by $\sigma_{I}:=\Delta_{I}(\sigma):=\operatorname{det}\left(\sigma_{i_{j}-i}\right)$.

1.8. Define $D_{t}:=\sum_{i \geq 0} D_{i} t^{i}: \bigwedge M_{n} \rightarrow \bigwedge M_{n}[[t]]$. The first equation of formula (3) can be equivalently written as $D_{t}(\mathbf{p} \wedge \mathbf{q})=D_{t} \mathbf{p} \wedge D_{t} \mathbf{q}$ (see [6]). Let $D_{t}^{-1}=\sum_{i>0}(-1)^{i} \bar{D}_{i} t^{i}$ be the formal inverse of $D_{t}$ seen as an element of $\operatorname{End}_{A}\left(\bigwedge M_{n}\right)[[t]]$. Then

$$
D_{t}^{-1}(\mathbf{p} \wedge \mathbf{q})=D_{t}^{-1} \mathbf{p} \wedge D_{t}^{-1} \mathbf{q}
$$

for each $\mathbf{p}, \mathbf{q} \in \bigwedge M_{n}$ (see [8]). A direct check shows that (4) implies

$$
\bar{D}_{h}(\mathbf{p} \wedge \mathbf{q})=\sum_{\left\{h_{1}, h_{2} \geq 0 \mid h_{1}+h_{2}=h\right\}} \bar{D}_{h_{1}} \mathbf{p} \wedge \bar{D}_{h_{2}} \mathbf{q},
$$

for each $h \geq 0$ and each $\mathbf{p}, \mathbf{q} \in \bigwedge M$. For future purposes we observe that the equality $D_{t} \circ D_{t}^{(-1)}=1$, holding in $\operatorname{End}_{A}\left(\bigwedge M_{n}\right)[[t]]$, implies that $\bar{D}_{h}=\Delta_{(2, \ldots, h+1)}(D)$. In particular $\bar{D}_{1}=D_{1}$. Recall that $h>k$ implies $\wedge^{k} M \subseteq \operatorname{ker} \bar{D}_{h}$ (see [8, Proposition 4.1]. 
1.9. Let $E^{\bullet}$ be any complete flag of $\mathbb{C}^{n}$. If $I \in \mathcal{I}_{n}^{k}$ and $\operatorname{wt}(I)=w$,

$$
\omega_{I}:=\int_{G(k, n)} \sigma_{1}^{k(n-k)-w} \cap \Omega_{I}=\int_{G(k, n)} \sigma_{1}^{k(n-k)-w} \sigma_{I} \cap[G(k, n)]
$$

is the degree of the Schubert variety $\Omega_{I}\left(E^{\bullet}\right)$ in the Plücker embedding of $G(k, n)$. If $J=I^{\tau}$ one defines

$$
\omega_{J}=\operatorname{sgn}(\tau) \cdot \omega_{I}
$$

It is known since Schubert [20] that

$$
\omega_{I}=\frac{(k(n-k)-w) ! \prod_{j<k}\left(i_{j}-i_{k}\right)}{\left(n-i_{1}\right) ! \cdots\left(n-i_{k}\right) !} .
$$

1.10. We denote by $\mathcal{A}^{*}\left(\bigwedge M_{n}\right)$ the commutative sub-algebra of $\operatorname{End}_{\mathbb{Z}}\left(\bigwedge M_{n}\right)$, image of the natural evaluation morphism $\operatorname{ev}_{D}:=\mathbb{Z}[\boldsymbol{T}] \rightarrow \operatorname{End}_{\mathbb{Z}}\left(\bigwedge M_{n}\right)$, sending $T_{i} \mapsto D_{i}$. There is an obvious restriction morphism $\rho_{k}: \mathcal{A}^{*}\left(\bigwedge M_{n}\right) \rightarrow \operatorname{End}_{\mathbb{Z}}\left(\bigwedge^{k} M_{n}\right)$ mapping $P(D) \in \mathcal{A}^{*}\left(\bigwedge M_{n}\right)$ to $\left.P(D)\right|_{\bigwedge^{k} M_{n}}$. Let $\mathcal{A}^{*}\left(\bigwedge^{k} M_{n}\right):=\operatorname{Im}\left(\rho_{k}\right)$. By [8], $\bigwedge^{k} M_{n}$ has a natural structure of free module of rank 1 over $\mathcal{A}^{*}\left(\bigwedge^{k} M_{n}\right)$, generated by $g_{k, n}$ (see 1.3$)$, as a consequence of Giambelli's formula:

$$
\epsilon^{i_{1}} \wedge \cdots \wedge \epsilon^{i_{k}}=\Delta_{\left(i_{1}, \ldots, i_{k}\right)}(D) \epsilon^{1} \wedge \cdots \wedge \epsilon^{k}
$$

Let $\hat{\Omega}_{k}: \bigwedge^{k} M_{n} \rightarrow A_{*}(G(k, n))$ be the obvious module isomorphism sending $\epsilon^{I} \mapsto \Omega_{I}$. In particular $\hat{\Omega}_{k}\left(g_{k, n}\right)=[G(k, n)]$, the fundamental class of $G(k, n)$, and $\hat{\Omega}_{k}\left(\pi_{k, n}\right)=\Omega_{n-k+1, \ldots, n}=[\mathrm{pt}]$, the class of a point. The main result of [6] implies that there is a ring isomorphism $\iota_{k}: A^{*}(G(k, n)) \rightarrow \mathcal{A}^{*}\left(\bigwedge^{k} M_{n}\right)$, sending $\sigma_{i} \mapsto D_{i}$, such that the diagram

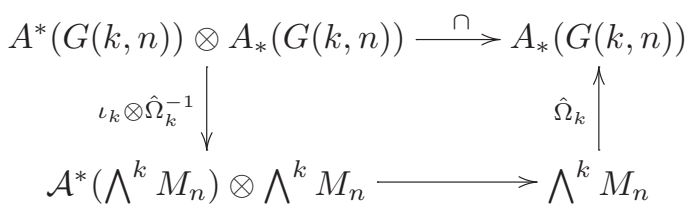

commutes, where the bottom horizontal map is defined by the module structure of $\bigwedge^{k} M_{n}$ over $\mathcal{A}^{*}\left(\bigwedge^{k} M_{n}\right)$. In particular, if $P \in \mathbb{Z}[\boldsymbol{T}]$ and $\sum_{J \in \mathcal{I}_{n}^{k}} a_{J} \cdot \boldsymbol{\epsilon}^{J}$ is the expansion of $P(D) \epsilon^{I}$ as an integral linear combination of the $\boldsymbol{\epsilon}^{I} \mathrm{~s}$, then

$$
P(\sigma) \cap \Omega_{I}=\hat{\Omega}_{k}\left(P(D) \epsilon^{I}\right)=\hat{\Omega}_{k}\left(\sum_{J \in \mathcal{I}_{n}^{k}} a_{J} \epsilon^{J}\right)=\sum_{J \in \mathcal{I}_{n}^{k}} a_{J} \hat{\Omega}_{k}\left(\epsilon^{J}\right)=\sum_{J \in \mathcal{I}_{n}^{k}} a_{J} \Omega_{J},
$$

where $P(\sigma) \in A^{*}(G(k, n))$ is the evaluation of $P$ at $\sigma$, via the map $T_{i} \mapsto \sigma_{i}$. Therefore,

$$
\int_{G(k, n)} P(\sigma) \cap \Omega_{I}=\int_{n} P(D) \cdot \epsilon^{I},
$$


where $\int_{G(k, n)} P(\sigma) \cap \Omega_{I}$ denotes the usual degree of the cycle $P(\sigma) \cap \Omega_{I} \in A_{*}(G(k, n))$, i.e., the coefficient of $[p t] \in A_{*}(G(k, n))$, the class of the point of $G(k, n)$, in the expansion of $P(\sigma) \cap \Omega_{I}$.

\section{Newton's formulas in Schubert Calculus}

2.1. The binomial coefficient $\left(\begin{array}{l}n \\ h\end{array}\right)$ is, by definition, the coefficient of $a^{h} b^{n-h}$ in the expansion of $(a+b)^{n}$. Therefore $\left(\begin{array}{l}n \\ j\end{array}\right)=0$ if $j<0, n<0$, or $j>n$. If $m, h \geq 0$ are integers, let $p_{h}(m)=\left\{\boldsymbol{\mu}:=\left(\mu_{1}, \ldots, \mu_{h}\right) \in \mathbb{N}^{h} \mid \sum_{i=1}^{h} m_{i}=m\right\}$. Denote by $|A|$ the cardinality of a set $A$ (we use boldface for multi-indices denoted by greek letters). Then (see [1, p. 33]),

$$
\left|p_{h}(m)\right|=\left(\begin{array}{c}
m+h-1 \\
h-1
\end{array}\right) .
$$

Define the multinomial coefficients via the equality

$$
\left(a_{1}+\cdots+a_{h}\right)^{m}=\sum_{\boldsymbol{\mu} \in p_{h}(m)}\left(\begin{array}{c}
m \\
\boldsymbol{\mu}
\end{array}\right) a_{1}^{\mu_{1}} \cdots a_{h}^{\mu_{h}} .
$$

With the usual convention $0 !=1$, the multinomial coefficient can be computed as

$$
\left(\begin{array}{c}
m \\
\boldsymbol{\mu}
\end{array}\right):=\frac{m !}{\mu_{1} ! \cdots \mu_{h} !}
$$

if $\boldsymbol{\mu} \in p_{h}(m)$, while is evidently zero otherwise. Equation (3) for $h=1$, implies:

Proposition 2.2. For each $\mathbf{p}, \mathbf{q} \in \bigwedge M_{n}$ and each $h \geq 0$, Newton's binomial formula holds:

$$
D_{1}^{m}(\mathbf{p} \wedge \mathbf{q})=\sum_{j=0}^{m}\left(\begin{array}{c}
m \\
j
\end{array}\right) D_{1}^{j} \mathbf{p} \wedge D_{1}^{m-j} \mathbf{q} .
$$

Proof. An obvious induction left to the reader.

Corollary 2.3. Let $m \geq 0$ and $\mathbf{p}_{1}, \ldots, \mathbf{p}_{h} \in \wedge M_{n}$. Then,

$$
D_{1}^{m}\left(\mathbf{p}_{1} \wedge \cdots \wedge \mathbf{p}_{h}\right)=\sum_{\boldsymbol{\mu} \in p_{h}(m)}\left(\begin{array}{c}
m \\
\boldsymbol{\mu}
\end{array}\right) D_{1}^{\mu_{1}} \mathbf{p}_{1} \wedge \cdots \wedge D_{1}^{\mu_{h}} \mathbf{p}_{h} .
$$

Proof. By induction on the integer $h \geq 2$. The case $h=2$ is Proposition 2.2. Suppose that the formula holds for $h-1$. Then, by Proposition 2.2,

$$
\begin{aligned}
D_{1}^{m}\left(\mathbf{p}_{1} \wedge \mathbf{p}_{2} \wedge \cdots \wedge \mathbf{p}_{h}\right) & =D_{1}^{m}\left(\mathbf{p}_{1} \wedge\left(\mathbf{p}_{2} \wedge \cdots \wedge \mathbf{p}_{h}\right)\right) \\
& =\sum_{m_{1}=0}^{m}\left(\begin{array}{c}
m \\
m_{1}
\end{array}\right) D_{1}^{m_{1}} \mathbf{p}_{1} \wedge D_{1}^{m-m_{1}}\left(\mathbf{p}_{2} \wedge \cdots \wedge \mathbf{p}_{h}\right) .
\end{aligned}
$$


By induction, (10) can be written as

$$
\begin{aligned}
& \sum_{m_{1}=0}^{m} \sum_{\left(m_{2}, \ldots, m_{h}\right) \in p_{h-1}\left(m-m_{1}\right)}\left(\begin{array}{c}
m \\
m_{1}
\end{array}\right) \frac{\left(m-m_{1}\right) !}{m_{2} ! \cdots m_{h} !} D_{1}^{m_{1}} \mathbf{p}_{1} \wedge D_{1}^{m_{2}} \mathbf{p}_{2} \wedge \cdots \wedge D_{1}^{m_{h}} \mathbf{p}_{h} \\
& =\sum_{\boldsymbol{\mu} \in p_{h}(m)}\left(\begin{array}{l}
m \\
\boldsymbol{\mu}
\end{array}\right) D_{1}^{\mu_{1}} \mathbf{p}_{1} \wedge \cdots \wedge D_{1}^{\mu_{h}} \mathbf{p}_{h} .
\end{aligned}
$$

Lemma 2.4. For each $i \geq 1, h \geq 0$, and $\mathbf{p} \in \bigwedge M$, the following formula holds:

$$
D_{h}\left(\epsilon^{i} \wedge \mathbf{p}\right)=\epsilon^{i} \wedge D_{h} \mathbf{p}+D_{h-1}\left(\epsilon^{i+1} \wedge \mathbf{p}\right)
$$

Proof. By a direct check, expanding the two sides of (11), according to Leibniz's rule (3).

Theorem 2.5. Let $h, m \geq 0, i \geq 1$ and $\mathbf{p} \in \bigwedge M_{n}$. Then,

$$
D_{h}^{m}\left(\epsilon^{i} \wedge \mathbf{p}\right)=\sum_{j=0}^{m}\left(\begin{array}{c}
m \\
j
\end{array}\right) D_{h-1}^{j}\left(\epsilon^{i+j} \wedge D_{h}^{m-j} \mathbf{p}\right)
$$

Proof. For $m=1$, formula (12) is (11). Suppose (12) holds for $m-1$. Since $D_{h}^{m}\left(\epsilon^{i} \wedge \mathbf{p}\right)=D_{h}\left(D_{h}^{m-1}\left(\epsilon^{i} \wedge \mathbf{p}\right)\right)$, induction on $m$ gives

$$
D_{h}^{m}\left(\epsilon^{i} \wedge \mathbf{p}\right)=D_{h}\left(\sum_{j=0}^{m-1}\left(\begin{array}{c}
m-1 \\
j
\end{array}\right) D_{h-1}^{j}\left(\epsilon^{i+j} \wedge D_{h}^{m-1-j} \mathbf{p}\right)\right)
$$

Using the linearity and the fact that the operators $\left\{D_{h}\right\}_{h \geq 0}$ are pairwise commuting,

$$
D_{h}^{m}\left(\epsilon^{i} \wedge \mathbf{p}\right)=\left(\sum_{j=0}^{m-1}\left(\begin{array}{c}
m-1 \\
j
\end{array}\right) D_{h-1}^{j} D_{h}\left(\epsilon^{i+j} \wedge D_{h}^{m-1-j} \mathbf{p}\right)\right)
$$

from which, by applying (11) again, one gets

$$
\begin{aligned}
D_{h}^{m}\left(\epsilon^{i} \wedge \mathbf{p}\right)= & \sum_{j=0}^{m-1}\left(\begin{array}{c}
m-1 \\
j
\end{array}\right) D_{h-1}^{j}\left(\epsilon^{i+j} \wedge D_{h}^{m-j} \mathbf{p}+D_{h-1}\left(\epsilon^{i+j+1} \wedge D_{h}^{m-1-j} \mathbf{p}\right)\right) \\
= & \sum_{j=0}^{m-1}\left(\begin{array}{c}
m-1 \\
j
\end{array}\right) D_{h-1}^{j}\left(\epsilon^{i+j} \wedge D_{h}^{m-j} \mathbf{p}\right) \\
& +\sum_{j=0}^{m-1}\left(\begin{array}{c}
m-1 \\
j
\end{array}\right) D_{h-1}^{j+1}\left(\epsilon^{i+j+1} \wedge D_{h}^{m-1-j} \mathbf{p}\right)=
\end{aligned}
$$




$$
\begin{aligned}
= & \sum_{j=0}^{m}\left(\begin{array}{c}
m-1 \\
j
\end{array}\right) D_{h-1}^{j}\left(\epsilon^{i+j} \wedge D_{h}^{m-j} \mathbf{p}\right) \\
& +\sum_{j=0}^{m}\left(\begin{array}{c}
m-1 \\
j-1
\end{array}\right) D_{h-1}^{j}\left(\epsilon^{i+j} \wedge D_{h}^{m-j} \mathbf{p}\right) \\
= & \sum_{j=0}^{m}\left[\left(\begin{array}{c}
m-1 \\
j
\end{array}\right)+\left(\begin{array}{c}
m-1 \\
j-1
\end{array}\right)\right] D_{h-1}^{j}\left(\epsilon^{i+j} \wedge D_{h}^{m-j} \mathbf{p}\right) \\
= & \sum_{j=0}^{m}\left(\begin{array}{c}
m \\
j
\end{array}\right) D_{h-1}^{j}\left(\epsilon^{i+j} \wedge D_{h}^{m-j} \mathbf{p}\right) .
\end{aligned}
$$

Theorem 2.6. Let $\mathbf{p} \in \bigwedge M_{n}$. Then for each $i \geq 1$ and each $h \geq 0$ one has (notation as in 1.8):

$$
\bar{D}_{h}^{m}\left(\epsilon^{i} \wedge \mathbf{p}\right)=\sum_{j=0}^{m}\left(\begin{array}{c}
m \\
j
\end{array}\right) \epsilon^{i+j} \wedge \bar{D}_{h-1}^{j} \bar{D}_{h}^{m-j} \mathbf{p}
$$

Proof. By induction on $m$. Recall that, by [8, Proposition 4.1], $\bar{D}_{j} \epsilon^{i}=0$ unless $0 \leq j \leq 1$, in which case one has $\bar{D}_{0} \epsilon^{i}=\epsilon^{i}$ and $\bar{D}_{1} \epsilon^{i}=D_{1} \epsilon^{i}=\epsilon^{i+1}$. Then,

$$
\bar{D}_{h}\left(\epsilon^{i} \wedge \mathbf{p}\right)=\sum_{j=0}^{h} \bar{D}_{j} \epsilon^{i} \wedge \bar{D}_{h-j} \mathbf{p}=\epsilon^{i} \wedge \bar{D}_{h} \mathbf{p}+\epsilon^{i+1} \wedge \bar{D}_{h-1} \mathbf{p}
$$

i.e., (13) holds for $m=1$. Suppose it holds for $m-1$. Then

$$
\bar{D}_{h}^{m}\left(\epsilon^{i} \wedge \mathbf{p}\right)=\bar{D}_{h}\left(\bar{D}_{h}^{m-1}\left(\epsilon^{i} \wedge \mathbf{p}\right)\right)=\bar{D}_{h} \sum_{j=0}^{m-1}\left(\begin{array}{c}
m-1 \\
j
\end{array}\right) \epsilon^{i+j} \wedge \bar{D}_{h-1}^{j} \bar{D}_{h}^{m-1-j} \mathbf{p}
$$

which is equal, by linearity and the definition of $\bar{D}_{h}$, to 


$$
\begin{aligned}
\sum_{j=0}^{m-1}\left(\begin{array}{c}
m-1 \\
j
\end{array}\right) \epsilon^{i+j} \wedge \bar{D}_{h-1}^{j} \bar{D}_{h}^{m-j} \mathbf{p} & +\sum_{j=0}^{m-1}\left(\begin{array}{c}
m-1 \\
j
\end{array}\right) \epsilon^{i+j+1} \wedge \bar{D}_{h-1}^{j+1} \bar{D}_{h}^{m-1-j} \mathbf{p} \\
= & \sum_{j=0}^{m}\left(\begin{array}{c}
m-1 \\
j
\end{array}\right) \epsilon^{i+j} \wedge \bar{D}_{h-1}^{j} \bar{D}_{h}^{m-j} \mathbf{p} \\
& +\sum_{j=0}^{m}\left(\begin{array}{c}
m-1 \\
j-1
\end{array}\right) \epsilon^{i+j} \wedge \bar{D}_{h-1}^{j} \bar{D}_{h}^{m-1} \mathbf{p} \\
= & \sum_{j=0}^{m}\left[\left(\begin{array}{c}
m-1 \\
j
\end{array}\right)-\left(\begin{array}{c}
m-1 \\
j-1
\end{array}\right)\right] \epsilon^{i+j} \wedge \bar{D}_{h-1}^{j} \bar{D}_{h}^{m-j} \mathbf{p} \\
= & \sum_{j=0}^{m}\left(\begin{array}{c}
m \\
j
\end{array}\right) \epsilon^{i+j} \wedge \bar{D}_{h-1}^{j} \bar{D}_{h}^{m-j} \mathbf{p},
\end{aligned}
$$

as claimed.

2.7. Formula (12) supports an explicit efficient algorithm to express any product of special Schubert cycles as an integral linear combination of products of the form $\sigma_{1}^{m} \cap \sigma_{I}$. Such an algorithm is extremely useful to perform computations, but it has also a theoretical relevance: it shows that the algebra structure of $A^{*}(G(k, n))$ is completely determined once one knows the product $\sigma_{1} \sigma_{I}$ for each $I \in \mathcal{I}_{n}^{k}$. To this purpose we exploit diagram (6) together with our main formula (12), as follows. For each $I:=\left(i_{1}, \ldots, i_{k}\right) \in \mathcal{I}_{n}^{k}, h \geq 1$, and $d, m \geq 0$, let

$$
\mathcal{J}(d, k, I):=\left\{(j, J) \in \mathbb{N} \times \mathcal{I}_{n}^{k} \mid j+\operatorname{wt}(J)=\operatorname{wt}(I)+d\right\} .
$$

Proposition 2.8. There is an explicit algorithm to express, in at least one way, any $D_{h}^{m}\left(\epsilon^{I}\right)$ as a $\mathbb{Z}$-linear combination of the elements of the set

$$
\left\{D_{1}^{j}\left(\boldsymbol{\epsilon}^{J}\right) \mid(j, J) \in \mathcal{J}(m h, k, I)\right\},
$$

i.e., $D_{h}^{m}\left(\boldsymbol{\epsilon}^{I}\right)=\sum_{(j, J) \in \mathcal{J}(m h, k, I)} a_{J} D_{1}^{j}\left(\boldsymbol{\epsilon}^{J}\right)$.

Proof. Induction on $h \geq 1$ and on $k \geq 2$. For $h=1$ the proposition is trivial for all $k \geq 2$. Let us assume that it holds for all $1 \leq h^{\prime} \leq h-1$, and all $\epsilon^{i_{1}} \wedge \epsilon^{i_{2}} \in \bigwedge^{2} M_{n}$ $(k=2)$. By $(12)$,

$$
D_{h}^{m}\left(\epsilon^{i_{1}} \wedge \epsilon^{i_{2}}\right)=\sum_{j=0}^{m}\left(\begin{array}{c}
m \\
j
\end{array}\right) D_{h-1}^{j}\left(\epsilon^{i_{1}+j} \wedge \epsilon^{i_{2}+h(m-j)}\right) .
$$

By the inductive hypothesis $D_{h-1}^{j}\left(\epsilon^{i_{1}+j} \wedge \epsilon^{i_{2}+h(m-j)}\right)$ is equal to a suitable linear combination of elements of the form $D_{1}^{j^{\prime}}\left(\epsilon^{j_{1}} \wedge \epsilon^{j_{2}}\right)$. Hence the proposition holds 
for $k=2$ and for all $h \geq 1$. Suppose now that the proposition holds (i) for all $1 \leq h^{\prime} \leq h-1$ and all $I \in \mathcal{I}_{n}^{k}(k \geq 2)$ and (ii) for all $h \geq 1$, and all $I^{\prime}:=\left(i_{2}, \ldots, i_{k}\right) \in \mathcal{I}_{n}^{k-1}$. We apply $(12)$ to $\epsilon^{i_{1}} \wedge \mathbf{p}$, with $\mathbf{p}:=\epsilon^{I^{\prime}}=\epsilon^{i_{2}} \wedge \cdots \wedge \epsilon^{i_{k}}$ :

$$
D_{h}^{m}\left(\epsilon^{i_{1}} \wedge \cdots \wedge \epsilon^{i_{k}}\right)=\sum_{j=0}^{m}\left(\begin{array}{c}
m \\
j
\end{array}\right) D_{h-1}^{j}\left(\epsilon^{i_{1}+j} \wedge D_{h}^{m-j}\left(\epsilon^{i_{2}} \wedge \cdots \wedge \epsilon^{i_{k}}\right)\right)
$$

By induction, $D_{h}^{m-j}\left(\epsilon^{i_{2}} \wedge \cdots \wedge \epsilon^{i_{k}}\right)$ can be written as

$$
\sum_{j=0}^{m} \sum_{\left(j^{\prime}, J^{\prime}\right) \in \mathcal{J}\left(h(m-j), k-1, I^{\prime}\right)} a_{J^{\prime}} D_{1}^{j^{\prime}}\left(\boldsymbol{\epsilon}^{J^{\prime}}\right),
$$

for a suitable choice of $a_{J^{\prime}} \in \mathbb{Z}$, and (15) takes the form

$$
D_{h}^{m}\left(\epsilon^{i_{1}} \wedge \cdots \wedge \epsilon^{i_{k}}\right)=\sum_{j=0}^{m}\left(\begin{array}{c}
m \\
j
\end{array}\right) D_{h-1}^{j}\left(\epsilon^{i_{1}+j} \wedge \sum_{\left(j^{\prime}, J^{\prime}\right) \in \mathcal{J}\left(h(m-j), k-1, I^{\prime}\right)} a_{J^{\prime}} D_{1}^{j^{\prime}}\left(\epsilon^{J^{\prime}}\right)\right) .
$$

By Corollary 2.3 one has

$$
D_{1}^{j^{\prime}}\left(\boldsymbol{\epsilon}^{J^{\prime}}\right)=\sum_{\left(l_{2}, \ldots, l_{k}\right) \in p_{k-1}\left(j^{\prime}\right)} \frac{j^{\prime} !}{l_{2} ! \cdots l_{k} !} \epsilon^{j_{2}^{\prime}+l_{2}} \wedge \cdots \wedge \epsilon^{j_{k}^{\prime}+l_{k}} .
$$

In conclusion,

$$
\begin{aligned}
D_{h}^{m}\left(\epsilon^{i_{1}} \wedge \cdots \wedge \epsilon^{i_{k}}\right) & \\
& =\sum_{j=0}^{m}\left(\begin{array}{c}
m \\
j
\end{array}\right) D_{h-1}^{j}\left(\sum_{\left(l_{2}, \ldots, l_{k}\right) \in p_{k-1}\left(j^{\prime}\right)} \frac{j^{\prime} !}{l_{2} ! \cdots l_{k} !} \epsilon^{i_{1}} \wedge \epsilon^{j_{2}^{\prime}+l_{2}} \wedge \cdots \wedge \epsilon^{j_{k}^{\prime}+l_{k}}\right) \\
& =\sum_{\substack{0 \leq j \leq m \\
\left(l_{2}, \ldots, l_{k}\right) \in p_{k-1}\left(j^{\prime}\right)}}\left(\begin{array}{c}
m \\
j
\end{array}\right) \frac{j^{\prime} !}{l_{2} ! \cdots l_{k} !} D_{h-1}^{j}\left(\epsilon^{i_{1}} \wedge \epsilon^{j_{2}^{\prime}+l_{2}} \wedge \cdots \wedge \epsilon^{j_{k}^{\prime}+l_{k}}\right) .
\end{aligned}
$$

By the inductive hypothesis, one then sees that $D_{h}^{m}\left(\epsilon^{i_{1}} \wedge \cdots \wedge \epsilon^{i_{k}}\right)$ is itself an integral linear combination of the elements of the set (14).

Corollary 2.9. Let $P \in \mathbb{Z}[\boldsymbol{T}]_{h}$ (see section 1.6). There is an explicit effective algorithm to express $P(D) \epsilon^{i_{1}} \wedge \cdots \wedge \epsilon^{i_{k}}$, in at least one way, as an integral linear combination of $\left\{D_{1}^{j}\left(\boldsymbol{\epsilon}^{J}\right)\right\}(j, J) \in \mathcal{J}(h, k, I)$.

Proof. Any such $P$ is, by definition, a (finite) integral linear combination of monomials of the form $T_{h_{1}}^{m_{1}} T_{h_{2}}^{m_{2}} \cdots T_{h_{l}}^{m_{l}}$, and then it suffices to check the property for any such 
a term. One argues by induction on the integer $l$ : For $l=1$ the property holds. If it holds for $l-1$, one has that

$$
D_{h_{1}}^{m_{1}} D_{h_{2}}^{m_{2}} \cdots D_{h_{l}}^{m_{l}}\left(\epsilon^{i_{1}} \wedge \cdots \wedge \epsilon^{i_{k}}\right)=D_{h_{1}}^{m_{1}}\left(D_{h_{2}}^{m_{2}} \cdots D_{h_{l}}^{m_{l}}\left(\epsilon^{i_{1}} \wedge \cdots \wedge \epsilon^{i_{k}}\right)\right)
$$

By induction, there are integers $a_{J}$ such that

$$
D_{h_{2}}^{m_{2}} \cdots D_{h_{l}}^{m_{l}}\left(\epsilon^{i_{1}} \wedge \cdots \wedge \epsilon^{i_{k}}\right)=\sum_{(j, J)} a_{J} D_{1}^{j} \epsilon^{J},
$$

the sum over all $(j, J)$ such that $j+\operatorname{wt}(J)=w t(I)+h_{2} m_{2}+\cdots+h_{l} m_{l}$. Hence,

$$
D_{h_{1}}^{m_{1}} D_{h_{2}}^{m_{2}} \cdots D_{h_{l}}^{m_{l}}\left(\epsilon^{i_{1}} \wedge \cdots \wedge \epsilon^{i_{k}}\right)=D_{h_{1}}^{m_{1}}\left(\sum_{(j, J)} a_{J} D_{1}^{j} \epsilon^{J}\right)=\sum_{(j, J)} a_{J} D_{1}^{j}\left(D_{h_{1}}^{m_{1}} \epsilon^{J}\right)
$$

and one finally concludes by applying Proposition 2.8 again.

Corollary 2.10. Suppose $\operatorname{deg}(P)=k(n-k)$. There is an explicit algorithm to compute

$$
\int_{G(k, n)} P(\sigma) \cap[G(k, n)]=\int_{n} P(D)\left(\epsilon^{1} \wedge \cdots \wedge \epsilon^{k}\right)
$$

as a $\mathbb{Z}$-linear combination of degrees of Schubert varieties.

Proof. In fact, by Corollary (2.9) one can determine integers $a_{J}$ to write

$$
P(D) \epsilon^{1} \wedge \cdots \wedge \epsilon^{k}=\sum_{(j, J)} a_{J} D_{1}^{j} \epsilon^{J},
$$

where in each summand $j+\operatorname{wt}(J)=k(n-k)$. Taking integrals,

$$
\int_{n} P(D) \epsilon^{1} \wedge \cdots \wedge \epsilon^{k}=\sum_{(j, J)} a_{J} \int_{n} D_{1}^{j} \epsilon^{J} .
$$

Since $j+\operatorname{wt}(J)=k(n-k), \int_{n} D_{1}^{j} \epsilon^{J}$ is precisely the degree of the Schubert variety $\Omega_{J}\left(E^{\bullet}\right), E^{\bullet}$ being an arbitrary complete flag of $\mathbb{C}^{n}$ (see sections 1.7, 1.9).

\section{Computations in $\bigwedge^{k} M_{n}, 2 \leq k \leq 4$}

By [4], Schubert calculus on $G(k, n+k)$ can be interpreted in terms of enumerative geometry of linear series on the projective line with prescribed ramification divisor. This fact motivates the computation below which shall be applied to enumerative problems regarding rational curves in next section. 
3.1. Notation as in section 1.8. Recall that, for each $h \geq 0$ (see [8, sec. 2.10]),

$$
\bar{D}_{h}\left(\epsilon^{i_{1}} \wedge \cdots \wedge \epsilon^{i_{h}}\right)=\epsilon^{i_{1}+1} \wedge \cdots \wedge \epsilon^{i_{h}+1},
$$

and hence, by induction,

$$
\bar{D}_{h}^{m}\left(\epsilon^{i_{1}} \wedge \cdots \wedge \epsilon^{i_{h}}\right)=\epsilon^{i_{1}+m} \wedge \cdots \wedge \epsilon^{i_{h}+m} .
$$

Proposition 3.2. For each $h \geq 1$,

$$
\bar{D}_{h-1}^{m}\left(\epsilon^{i_{1}} \wedge \cdots \wedge \epsilon^{i_{h}}\right)=\sum_{\boldsymbol{\mu} \in p_{h}(m)}\left(\begin{array}{l}
m \\
\boldsymbol{\mu}
\end{array}\right) \epsilon^{i_{1}+\sum_{j \neq 1} \mu_{j}} \wedge \cdots \wedge \epsilon^{i_{h}+\sum_{j \neq h} \mu_{j}}
$$

Proof. If $h=1, D_{0} \epsilon^{i_{1}}=\epsilon^{i_{1}}$ and the proposition is trivial. For $h=2$, is just Newton formula (9), since $\bar{D}_{1}=D_{1}$ and

$$
D_{1}^{m}\left(\epsilon^{i_{1}} \wedge \epsilon^{i_{2}}\right)=\sum_{m_{1}=0}^{m}\left(\begin{array}{c}
m \\
m_{1}
\end{array}\right) \epsilon^{i_{1}+m_{1}} \wedge \epsilon^{i_{2}+m-m_{1}}=\sum_{\boldsymbol{\mu} \in p_{2}(m)}\left(\begin{array}{l}
m \\
\boldsymbol{\mu}
\end{array}\right) \epsilon^{i_{1}+\mu_{1}} \wedge \epsilon^{i_{2}+\mu_{2}} .
$$

Suppose the formula true for $h-1$. Then

$$
\bar{D}_{h-1}^{m}\left(\epsilon^{i_{1}} \wedge \cdots \wedge \epsilon^{i_{h}}\right)=\bar{D}_{h-1}^{m}\left(\epsilon^{i_{1}} \wedge\left(\epsilon^{i_{2}} \wedge \cdots \wedge \epsilon^{i_{h}}\right)\right),
$$

which by (13) is equal to

$$
\begin{aligned}
\sum_{m_{1}=0}^{m}\left(\begin{array}{c}
m \\
m_{1}
\end{array}\right) \epsilon^{i_{1}+m_{1}} & \wedge \bar{D}_{h-2}^{m_{1}}\left(\epsilon^{i_{2}+m-m_{1}} \wedge \cdots \wedge \epsilon^{i_{h}+m-m_{1}}\right) \\
= & \sum\left(\begin{array}{c}
m \\
m_{1}
\end{array}\right) \frac{m_{1} !}{m_{2} ! \cdots m_{h-1} !} \\
& \times \epsilon^{i_{1}+m_{1}} \wedge \epsilon^{i_{2}+m-m_{1}+\sum_{j \neq 2} m_{j}} \wedge \cdots \wedge \epsilon^{i_{h}+m-m_{1}+\sum_{j \neq h} m_{j}} \\
= & \sum \frac{m !}{\left(m-m_{1}\right) ! \cdot m_{2} ! \cdots m_{h} !} \\
& \times \epsilon^{i_{1}+m_{1}} \wedge \epsilon^{i_{2}+m-m_{1}+\sum_{j \neq 2} m_{j}} \wedge \cdots \wedge \epsilon^{i_{h}+m-m_{1}+\sum_{j \neq h} m_{j}},
\end{aligned}
$$

where the last two sums are over all $\left(m_{1}, m_{2}, \ldots, m_{h}\right)$ such that $0 \leq m_{1} \leq m$ and $\sum_{j=2}^{h} m_{j}=m_{1}$. Taking $\boldsymbol{\mu} \in p_{h}(m)$ such that $\mu_{1}=m-m_{1}$ and $\mu_{j}=m_{j}$, for $2 \leq j \leq h$, so that $\mu_{1}=m_{2}+\cdots+m_{h}$, one gets exactly formula (17).

Recall the notation of section 1.1.

Proposition 3.3. Let $n \geq 0$ and $M_{n}$ as in 1.1 and $D:=\left(D_{1}, D_{2}, \ldots\right)$ as in formula (3). Then the following equalities holds in $\wedge M_{n}$ : 


$$
D_{2}^{m}\left(\epsilon^{i_{1}} \wedge \epsilon^{i_{2}} \wedge \epsilon^{i_{3}}\right)=\sum_{\boldsymbol{\mu} \in p_{4}(m)}\left(\begin{array}{c}
m \\
\boldsymbol{\mu}
\end{array}\right) D_{1}^{\mu_{1}}\left(\epsilon^{i_{1}+\mu_{1}} \wedge \epsilon^{i_{2}+\mu_{2}+2 \mu_{4}} \wedge \epsilon^{i_{3}+2 \mu_{3}+\mu_{2}}\right) ;
$$

(ii)

$$
D_{2}^{m}\left(\epsilon^{i_{1}} \wedge \epsilon^{i_{2}} \wedge \epsilon^{i_{3}} \wedge \epsilon^{i_{4}}\right)=\sum_{\substack{\boldsymbol{\mu} \in p_{5}(m) \\
0 \leq l \leq \mu_{2}+\mu_{3}}}\left(\begin{array}{c}
m \\
\boldsymbol{\mu}
\end{array}\right)\left(\begin{array}{c}
\mu_{2}+\mu_{3} \\
l
\end{array}\right) D_{1}^{\mu_{1}}\left(\boldsymbol{\epsilon}^{I(\boldsymbol{\mu}, l)}\right),
$$

where

$$
I(\boldsymbol{\mu}, l)=\left(i_{1}+\mu_{1}, i_{2}+\mu_{2}+2 \mu_{5}, i_{3}+\mu_{3}+l, i_{4}+\mu_{2}+2 \mu_{4}+\mu_{3}-l\right) ;
$$

$$
\bar{D}_{2}^{m}\left(\epsilon^{i_{1}} \wedge \epsilon^{i_{2}} \wedge \epsilon^{i_{3}} \wedge \epsilon^{i_{4}}\right)=\sum_{\substack{\boldsymbol{\mu} \in p_{4}(m) \\
0 \leq l \leq \mu_{1}+\mu_{2}}}\left(\begin{array}{c}
m \\
\boldsymbol{\mu}
\end{array}\right)\left(\begin{array}{c}
\mu_{1}+\mu_{2} \\
l
\end{array}\right) \epsilon^{J(\boldsymbol{\mu}, l)},
$$

where

$$
J(\boldsymbol{\mu}, l):=\left(i_{1}+\mu_{1}+\mu_{4}, i_{2}+\mu_{2}+\mu_{4}, i_{3}+\mu_{3}+l, i_{4}+\mu_{1}+\mu_{2}+\mu_{3}-l\right) .
$$

Proof. It consists in a repeated application of (12). We limit ourselves to the verification of (ii) and (iii), leaving the easier (i) to the reader's care, as a more or less amusing exercise.

Regarding formula (ii) we first observe that

$$
\begin{aligned}
& D_{2}^{m}\left(\epsilon^{i_{1}} \wedge \epsilon^{i_{2}} \wedge \epsilon^{i_{3}} \wedge \epsilon^{i_{4}}\right) \\
& =\sum_{\alpha_{1}=0}^{m}\left(\begin{array}{c}
m \\
\alpha_{1}
\end{array}\right) D_{1}^{\alpha_{1}}\left(\epsilon^{i_{1}+\alpha_{1}} \wedge D_{2}^{m-\alpha_{1}}\left(\epsilon^{i_{2}} \wedge \epsilon^{i_{3}} \wedge \epsilon^{i_{4}}\right)\right) \\
& =\sum_{\substack{0 \leq a_{1} \leq m \\
0 \leq a_{2} \leq m-\alpha_{1}}}^{m}\left(\begin{array}{c}
m \\
\alpha_{1}
\end{array}\right)\left(\begin{array}{c}
m-\alpha_{1} \\
a_{2}
\end{array}\right) D_{1}^{\alpha_{1}}\left(\epsilon^{i_{1}+\alpha_{1}} \wedge D_{1}^{\alpha_{2}}\left(\epsilon^{i_{2}+\alpha_{2}} \wedge D_{2}^{m-\alpha_{1}-\alpha_{2}}\left(\epsilon^{i_{3}} \wedge \epsilon^{i_{4}}\right)\right)\right) \\
& =\sum_{\boldsymbol{\alpha} \in p_{4}(m)}\left(\begin{array}{c}
m \\
\boldsymbol{\alpha}
\end{array}\right) D_{1}^{\alpha_{1}}\left(\epsilon^{i_{1}+\alpha_{1}} \wedge D_{1}^{\alpha_{2}}\left(\epsilon^{i_{2}+\alpha_{2}} \wedge D_{1}^{\alpha_{3}}\left(\epsilon^{i_{3}+\alpha_{3}} \wedge \epsilon^{i_{4}+2 \alpha_{4}}\right)\right)\right)
\end{aligned}
$$

where (18) has been gotten by repeatedly applying (12) and having set $\boldsymbol{\alpha}=$ $\left(\alpha_{1}, \alpha_{2}, \alpha_{3}, \alpha_{4}\right)$. Now, by (9),

$$
\begin{aligned}
D_{1}^{\alpha_{2}}\left(\epsilon ^ { i _ { 2 } + \alpha _ { 2 } } \wedge D _ { 1 } ^ { \alpha _ { 3 } } \left(\epsilon^{i_{3}+\alpha_{3}}\right.\right. & \left.\left.\wedge \epsilon^{i_{4}+2 \alpha_{4}}\right)\right) \\
& =\sum_{0 \leq b \leq \alpha_{2}}\left(\begin{array}{c}
\alpha_{2} \\
b
\end{array}\right) \epsilon^{i_{2}+\alpha_{2}+b} \wedge D_{1}^{\alpha_{3}+\alpha_{2}-b}\left(\epsilon^{i_{3}+\alpha_{3}} \wedge \epsilon^{i_{4}+2 \alpha_{4}}\right) .
\end{aligned}
$$

By definition of $D_{1}$ and by applying (9), once more,

$$
\sum_{\substack{0 \leq b \leq \alpha_{2} \\
0 \leq l \leq \alpha_{3}+\alpha_{2}-b}}\left(\begin{array}{c}
\alpha_{2} \\
b
\end{array}\right)\left(\begin{array}{c}
\alpha_{3}+\alpha_{2}-b \\
l
\end{array}\right) \cdot \boldsymbol{\epsilon}^{J_{1}(\boldsymbol{\alpha}, b, l)}
$$


with $J_{1}(\boldsymbol{\alpha}, b, l):=\left(i_{2}+\alpha_{2}+b, i_{3}+\alpha_{3}+l, i_{4}+2 \alpha_{4}+\alpha_{2}+\alpha_{3}-b-l\right)$.

Last expression plugged into (18) gives

$$
\sum_{\substack{\boldsymbol{\alpha} \in p_{4}(m) \\
0 \leq b \leq \alpha_{2} \\
0 \leq l \leq \alpha_{3}+\alpha_{2}-b}}\left(\begin{array}{c}
m \\
\boldsymbol{\alpha}
\end{array}\right)\left(\begin{array}{c}
\alpha_{2} \\
b
\end{array}\right)\left(\begin{array}{c}
\alpha_{3}+\alpha_{2}-b \\
l
\end{array}\right) \cdot D_{1}^{\alpha_{1}} \boldsymbol{\epsilon}^{J_{2}(\boldsymbol{\alpha}, b, l)},
$$

where $J_{2}(\boldsymbol{\alpha}, b, l):=\left(i_{1}+\alpha_{1}, i_{2}+\alpha_{2}+b, i_{3}+\alpha_{3}+l, i_{4}+2 \alpha_{4}+\alpha_{2}+\alpha_{3}-b-l\right)$, i.e., using the definition of the multinomial coefficient,

$$
\sum \frac{m !}{\alpha_{1} !\left(\alpha_{2}-b\right) ! \alpha_{3} ! \alpha_{4} ! b !}\left(\begin{array}{c}
\alpha_{3}+\alpha_{2}-b \\
l
\end{array}\right) \cdot D_{1}^{\alpha_{1}}\left(\boldsymbol{\epsilon}^{J_{2}(\boldsymbol{\alpha}, b, l)}\right) .
$$

We may rename the variables defining $\boldsymbol{\mu} \in p_{5}(m)$ as (the way to do that is not unique) $\mu_{1}:=\alpha_{1}, \mu_{2}=\alpha_{2}-b, \mu_{3}=\alpha_{3}, \mu_{4}=\alpha_{4}, \mu_{5}=b$. Finally, expressing $\boldsymbol{\alpha}, b$ as functions of such a $\boldsymbol{\mu} \in p_{5}(m)$, and substituting in (19), one gets

$$
\sum_{\substack{\boldsymbol{\mu} \in p_{5}(m) \\
0 \leq l \leq \mu_{2}+\mu_{3}}}\left(\begin{array}{c}
m \\
\boldsymbol{\mu}
\end{array}\right)\left(\begin{array}{c}
\mu_{2}+\mu_{3} \\
l
\end{array}\right) D_{1}^{\mu_{1}}\left(\boldsymbol{\epsilon}^{I(\boldsymbol{\mu}, l)}\right),
$$

having set $I(\boldsymbol{\mu}, l)=\left(i_{1}+\mu_{1}, i_{2}+\mu_{2}+2 \mu_{5}, i_{3}+\mu_{3}+l, i_{4}+\mu_{2}+2 \mu_{4}+\mu_{3}-l\right)$, which is precisely (ii).

We come now to the proof of (iii). By applying formula (13) once, one gets

$$
\bar{D}_{2}^{m}\left(\epsilon^{i_{1}} \wedge \epsilon^{i_{2}} \wedge \epsilon^{i_{3}} \wedge \epsilon^{i_{4}}\right)=\sum_{\alpha_{1}=0}^{m}\left(\begin{array}{c}
m \\
\alpha_{1}
\end{array}\right) \epsilon^{i_{1}+\alpha_{1}} \wedge \bar{D}_{1}^{\alpha_{1}} \bar{D}_{2}^{m-\alpha_{1}}\left(\epsilon^{i_{2}} \wedge \epsilon^{i_{3}} \wedge \epsilon^{i_{4}}\right),
$$

which is, again,

$$
\begin{gathered}
\sum_{\substack{0 \leq \alpha_{1} \leq m \\
0 \leq \alpha_{2} \leq m-\alpha_{1}}}\left(\begin{array}{c}
m \\
\alpha_{1}
\end{array}\right)\left(\begin{array}{c}
m-\alpha_{1} \\
\alpha_{2}
\end{array}\right) \epsilon^{i_{1}+\alpha_{1}} \wedge \bar{D}_{1}^{\alpha_{1}}\left(\epsilon^{i_{2}+\alpha_{2}} \wedge \bar{D}_{1}^{\alpha_{2}} \bar{D}_{2}^{m-\alpha_{1}-\alpha_{2}}\left(\epsilon^{i_{3}} \wedge \epsilon^{i_{4}}\right)\right) \\
=\sum_{\substack{\alpha_{i} \geq 0 \\
\alpha_{1}+\alpha_{2}+\alpha_{3}=m}} \frac{m !}{\alpha_{1} ! \alpha_{2} ! \alpha_{3} !} \epsilon^{i_{1}+\alpha_{1}} \wedge \bar{D}_{1}^{\alpha_{1}}\left(\epsilon^{i_{2}+\alpha_{2}} \wedge \bar{D}_{1}^{\alpha_{2}}\left(\epsilon^{i_{3}+\alpha_{3}} \wedge \epsilon^{i_{4}+\alpha_{3}}\right)\right)
\end{gathered}
$$

where in the last equality we used (8) (having put $\alpha_{3}:=m-\alpha_{1}-\alpha_{2}$ ) and (16). By 
applying twice formula (9) to expression (20) one gets

$$
\begin{gathered}
\sum_{\substack{\alpha_{1}+\alpha_{2}+\alpha_{3}=m \\
0 \leq b \leq \alpha_{1}}} \frac{m !}{\alpha_{1} ! \alpha_{2} ! \alpha_{3} !}\left(\begin{array}{c}
\alpha_{1} \\
b
\end{array}\right) \epsilon^{i_{1}+\alpha_{1}} \wedge \epsilon^{i_{2}+\alpha_{2}+b} \wedge \bar{D}_{1}^{\alpha_{2}+\alpha_{1}-b}\left(\epsilon^{i_{3}+\alpha_{3}} \wedge \epsilon^{i_{4}+\alpha_{3}}\right) \\
=\sum_{\substack{\alpha_{1}+\alpha_{2}+\alpha_{3}=m \\
0 \leq b \leq \alpha_{1} \\
0 \leq l \leq \alpha_{2}+\alpha_{1}-b}} \frac{m !}{\alpha_{1} ! \alpha_{2} ! \alpha_{3} !}\left(\begin{array}{c}
\alpha_{1} \\
b
\end{array}\right)\left(\begin{array}{c}
\alpha_{2}+\alpha_{1}-b \\
l
\end{array}\right) \\
\quad \times \epsilon^{i_{1}+\alpha_{1}} \wedge \epsilon^{i_{2}+\alpha_{2}+b} \wedge \epsilon^{i_{3}+\alpha_{3}+l} \wedge \epsilon^{i_{4}+\alpha_{3}+\alpha_{1}+\alpha_{2}-b-l} \\
=\sum_{\substack{\alpha_{1}+\alpha_{2}+\alpha_{3}=m \\
0 \leq b \leq \alpha_{1} \\
0 \leq l \leq \alpha_{2}+\alpha_{1}-b}} \frac{m !}{\left(\alpha_{1}-b\right) ! \alpha_{2} ! \alpha_{3} ! b !}\left(\begin{array}{c}
\alpha_{2}+\alpha_{1}-b \\
l
\end{array}\right) \\
\quad \times \epsilon^{i_{1}+\alpha_{1}} \wedge \epsilon^{i_{2}+\alpha_{2}+b} \wedge \epsilon^{i_{3}+\alpha_{3}+l} \wedge \epsilon^{i_{4}+\alpha_{3}+\alpha_{1}+\alpha_{2}-b-l} .
\end{gathered}
$$

Putting $\boldsymbol{\mu} \in p_{4}(m)$ such that $\mu_{1}=\alpha_{1}-b, \mu_{2}=\alpha_{2}, \mu_{3}=\alpha_{3}$, and $\mu_{4}=b$, one finally obtains (iii), as desired.

\section{Counting $g_{n+r}^{r}$ on $\mathbb{P}^{1}$ with prescribed ramification at distinct points.}

The main references for next subsection are [4] and [12, section 1].

4.1. If $a$ is a non-negative integer and $W$ a subspace of $F:=H^{0}\left(\mathbb{P}^{1}, O_{\mathbb{P}^{1}}(d)\right)$, denote by $W(-a P)$ the subspace of all the sections of $W$ vanishing at $P$ with multiplicity at least $a$. A $g_{d}^{r}$ on $\mathbb{P}^{1}$ is the choice of a $(r+1)$-dimensional subspace $V$ of $F$. Since $F(-a P)$ has codimension $a$, for each $0 \leq a \leq d+1$, the chain of inclusions

$$
F^{\bullet}(P): F \supset F(-P) \supset \cdots \supset F(-d P) \supset F(-(d+1) P)=0,
$$

defines the (complete) osculating flag of $F$ at $P$. If there exists a non zero $v \in V$ vanishing at $P$ with multiplicity exactly $i-1(i \geq 1)$, one says that $i-1$ is a $V$-order at $P$. There are exactly $r+1$ orders at each point $P$, forming its $V$-order sequence $0 \leq i_{1}-1<\cdots<i_{r+1}-1 \leq d$. Following [12, section 1.2], we shall say that $1 \leq i_{1}<\cdots<i_{r+1} \leq d+1$ is the $V$-ramification sequence at $P$. The $V$-weight of $P$ is $\operatorname{wt}_{V}(P)=\sum_{j=1}^{r+1}\left(i_{j}(P)-j\right)$. A point $P \in \mathbb{P}^{1}$ is a $V$-ramification point if one of the following equivalent conditions occur: (i) $\operatorname{dim} V(-(r+1) P)>1$, (ii) $\operatorname{wt}_{V}(P)>0$, (iii) the $V$-ramification sequence at $P$ is different from $(1,2,3, \ldots, r+1)$. All but finitely many points have weight 0 and the total weight of the ramification points is prescribed by the Brill-Segre-Plücker formula:

$$
\mathrm{wt}_{V}=\sum_{P \in C} \mathrm{wt}_{V}(P)=(r+1)(d-r) .
$$


4.2. If $\left(i_{1}(P), \ldots, i_{r+1}(P)\right)$ is the $V$-ramification sequence at $P$, then $V$ belongs, by [4], to the Schubert variety $\Omega_{i_{1}, \ldots, i_{r+1}}\left(F^{\bullet}(P)\right)$ of the Grassmannian $G(r+1, d+1)$. Moreover, if $P_{1}, \ldots, P_{m}$ are $m$ distinct points and $I_{1}, \ldots, I_{m} \in \mathcal{I}_{d}^{r+1}$, the Schubert varieties $\Omega_{I_{j}}\left(F^{\bullet}\left(P_{j}\right)\right)$ are dimensionally transverse, i.e., every irreducible component of $\bigcap_{j=1}^{m} \Omega_{I_{j}}\left(F^{\bullet}\left(P_{j}\right)\right)$ has codimension $\sum \operatorname{codim} \Omega_{I_{j}}\left(F^{\bullet}\left(P_{j}\right)\right)=\sum_{j=1}^{m} \mathrm{wt}\left(I_{j}\right)$. In particular, if the intersection is zero dimensional, the degree of the intersection cycle is given by the integral of the product of the corresponding Schubert cycles, in the intersection ring of $G(r+1, d+1)$, capped with the fundamental class of $G(r+1, d+1)$. The formulas gotten in section 3 via (12) can be easily applied to get expressions for the number (with multiplicity) of $g_{n+r}^{r}$ on $\mathbb{P}^{1}$ with prescribed ramifications at prescribed points for $r=1,2,3$, i.e., for pencils, nets, and webs.

4.3 Counting pencils on $\mathbb{P}^{1}$. The enumerative geometry of pencils on $\mathbb{P}^{1}$ with prescribed ramifications is ruled by the intersection theory on $G(2, n+2)$. By [8], $A^{*}(G(2, n+2))$ is generated by $D_{1}, D_{2}$.

Proposition 4.4. Let $a, b \geq 0$ and $i_{1}, i_{2} \geq 1$ such that $a+2 b=2 n-i_{1}-i_{2}-3$. Then,

$$
\int_{n+2} D_{1}^{a} D_{2}^{b}\left(\epsilon^{i_{1}} \wedge \epsilon^{i_{2}}\right)=\sum_{\beta=0}^{b}\left(\begin{array}{l}
b \\
\beta
\end{array}\right) \omega_{i_{1}+\beta, i_{2}+2 b-2 \beta} .
$$

Proof. In fact,

$$
\begin{aligned}
D_{1}^{a} D_{2}^{b}\left(\epsilon^{i_{1}} \wedge \epsilon^{i_{2}}\right) & =D_{1}^{a} \sum_{\beta=0}^{b}\left(\begin{array}{l}
b \\
\beta
\end{array}\right) D_{1}^{\beta}\left(\epsilon^{i_{1}+\beta} \wedge D_{2}^{b-\beta} \epsilon^{i_{2}}\right) \\
& =\sum_{\beta=0}^{b}\left(\begin{array}{l}
b \\
\beta
\end{array}\right) D_{1}^{a+\beta}\left(\epsilon^{i_{1}+\beta} \wedge \epsilon^{i_{2}+2 b-2 \beta}\right) .
\end{aligned}
$$

Taking integrals one obtains precisely formula (21).

$$
\begin{aligned}
& \text { If } i_{1}=1, i_{2}=2 \text { and } b=0 \text { (i.e., } a=2 n \text { ), } \\
& \qquad \int_{n+2} D_{1}^{2 n}\left(\epsilon^{1} \wedge \epsilon^{2}\right)=\omega_{1,2}=\left(\begin{array}{c}
2 n \\
n
\end{array}\right)-\left(\begin{array}{c}
2 n \\
n+1
\end{array}\right)=\frac{1}{n+1}\left(\begin{array}{c}
2 n \\
n
\end{array}\right),
\end{aligned}
$$

the degree of the Grassmannian $G(2, n+2)$, called Goldberg's formula in [16]. Let now $q_{1}, \ldots, q_{h}$ be non negative integers not bigger than $n$, such that $\sum q_{j}=2 n$.

Theorem 4.5 (Scherbak [18]). The following formula holds:

$$
\begin{aligned}
\int_{G(2, n+2)} \sigma_{q_{1}} \cdots \sigma_{q_{h}} \cap[G(2, n+2)] & \\
& =\sum_{I \subseteq\{1, \ldots, h\}}(-1)^{h+1-|I|}\left(\begin{array}{c}
\sum_{i \in I} q_{i}+|I|-n-1 \\
h-2
\end{array}\right) .
\end{aligned}
$$


Proof. Notation as in section 2.1. Diagram (6) guarantees the following equality:

$$
\int_{G(2, n+2)} \sigma_{q_{1}} \cdots \sigma_{q_{h}} \cap[G(2, n+2)]=\int_{n+2} D_{q_{1}} \cdots D_{q_{h}}\left(\epsilon^{1} \wedge \epsilon^{2}\right),
$$

where the last hand side is the coefficient of $\epsilon^{n+1} \wedge \epsilon^{n+2}$ in the expansion of $D_{q_{1}} \cdots D_{q_{h}}\left(\epsilon^{1} \wedge \epsilon^{2}\right)$. Iterating formula (3), one easily gets

$$
\begin{aligned}
D_{q_{1}} \cdots D_{q_{h}}\left(\epsilon^{1} \wedge \epsilon^{2}\right) & =\sum_{\substack{1 \leq j \leq h \\
0 \leq m_{j} \leq q_{j}}} \epsilon^{1+m_{1}+\cdots+m_{h}} \wedge \epsilon^{2+q_{1}+\cdots+q_{h}-m_{1}-\cdots-m_{h}} \\
& =\sum_{\substack{1 \leq j \leq h \\
0 \leq m_{j} \leq q_{j}}} \epsilon^{1+m_{1}+\cdots+m_{h}} \wedge \epsilon^{2+2 n-m_{1}-\cdots-m_{h}} .
\end{aligned}
$$

Since $\sum_{1<j<h} q_{j}=2 n$, the only surviving terms in the sum (23) are those for which either $m_{1}+\cdots+m_{h}=n$ or $m_{1}+\cdots+m_{h}=n+1$. For each $m \geq 0, h \geq 1$, and $1 \leq j \leq h$, let $p_{h ; j}(m):=\left\{\left(m_{1}, \ldots, m_{h}\right) \in p_{h}(m) \mid m_{j} \leq q_{j}\right\}$. Then,

$$
\int_{n+2} D_{q_{1}} \cdots D_{q_{h}}\left(\epsilon^{1} \wedge \epsilon^{2}\right)=\left|\bigcap_{1 \leq j \leq h} p_{h ; j}(n)\right|-\left|\bigcap_{1 \leq j \leq h} p_{h ; j}(n+1)\right|,
$$

and our task consists now in evaluating the right hand side. As done in [19], in the context of representation theory, we apply the inclusion-exclusion formula (see, e.g., $[1$, p. 76]) to our situation. First, one notices that for each $1 \leq j \leq h$, the set $p_{h ; j}(m)$ is the complement in $p_{h}(m)$ of

$$
p_{h ; j}^{\prime}(m):=\left\{\left(m_{1}, \ldots, m_{h}\right) \in p_{h}(m) \mid m_{j} \geq q_{j}+1\right\} .
$$

Therefore,

$$
\left|\bigcap_{1 \leq j \leq h} p_{h ; j}(m)\right|=\left|p_{h}(m)-\bigcup_{1 \leq j \leq h} p_{h ; j}^{\prime}(m)\right|=\sum_{J \subseteq\{1, \ldots, h\}}(-1)^{|J|}\left|\bigcap_{j \in J} p_{h ; j}^{\prime}(m)\right|,
$$

where we used De Morgan's laws and, for the second equality, the inclusion exclusion formula, with the convention that $\left|\bigcap_{j \in J} p_{h ; j}^{\prime}(m)\right|=\left|p_{h}(m)\right|$, if $|J|=\emptyset$. Now, for each $\left(m_{1}, \ldots, m_{h}\right) \in \bigcap_{j \in J} p_{h ; j}^{\prime}(m)$ and each $j \in J$, replace the element $m_{j}$ with $m_{j}^{\prime}=m_{j}-q_{j}-1$ to see that $\bigcap_{j \in J} p_{h ; j}^{\prime}(m)=p_{h}\left(m-\sum_{j \in J} q_{j}-|I|\right)$. Then,

$$
\left|\bigcap_{j \in J} p_{h ; j}^{\prime}(m)\right|=\left|p_{h}\left(m-\sum_{j \in J} q_{j}-|J|\right)\right|=\left(\begin{array}{c}
m-\sum_{j \in J} q_{j}-|J|+h-1 \\
h-1
\end{array}\right),
$$

where to get last equality in (24) we applied formula (7). 
Finally,

$$
\begin{aligned}
\int_{n+2} D_{q_{1}} \cdots D_{q_{h}}\left(\epsilon^{1} \wedge \epsilon^{2}\right)= & \left|\bigcap_{1 \leq j \leq h} p_{h ; j}(n)\right|-\left|\bigcap_{1 \leq j \leq h} p_{h ; j}(n+1)\right| \\
= & \sum_{J \subseteq\{1, \ldots, h\}}(-1)^{|J|}\left[\left(\begin{array}{c}
n-\sum_{j \in J} q_{j}-|J|+h-1 \\
h-1
\end{array}\right)\right. \\
& \left.-\left(\begin{array}{c}
n+1-\sum_{j \in J} q_{j}-|J|+h-1 \\
h-1
\end{array}\right)\right] \\
= & -\sum_{J \subseteq\{1, \ldots, h\}}(-1)^{|J|}\left(\begin{array}{c}
n-\sum_{j \in J} q_{j}-|J|+h-1 \\
h-2
\end{array}\right) \\
= & -\sum_{I \subseteq\{1, \ldots, h\}}(-1)^{h-|I|}\left(\begin{array}{c}
\sum_{i \in I} q_{i}+|I|-n-1 \\
h-2
\end{array}\right),
\end{aligned}
$$

where, for $I=\{1, \ldots, h\} \backslash J$, we have used the equality $\sum_{i \in I} q_{i}=2 n-\sum_{j \in J} q_{j}$. The last term of the equalities above obviously coincides with the r.h.s. of (22).

Remark 4.6. Expression (22) is Scherbak's formula in [18], written in a slightly modified version to (formally) include the cases $h=1$ - the degree (equal to 0 ) of a special Schubert cycle - and $h=2$ - the degree of the product of two special Schubert cycles.

4.7 Counting nets on $\mathbb{P}^{\mathbf{1}}$. When $r=2$, let $C$ be the image of $\mathbb{P}^{1}$ through the rational map $\phi: \mathbb{P}^{1} \rightarrow \mathbb{P}(V)$ induced the given $g_{d}^{2}$. Assume that $\phi$ is a morphism, i.e., the $g_{d}^{2}$ has no base point. The geometrical interpretation of the $V$-ramification sequence at a point $P$ in terms of the nature of the point $Q:=\phi(P)$ on $C$ is as follows. At a general point of $\mathbb{P}^{1}$, the $V$-ramification sequence is $(1,2,3)$ (weight 0 ) and $Q$ is an ordinary point of $C$. Instead, $Q$ is a flex, a hyperflex, a cusp, or a tacnode if the $V$-ramification sequence at $P$ is respectively $(1,2,4),(1,2,5),(1,3,4),(1,3,5)$. The $V$-weights are respectively $1,2,2$, and 3 . Suppose that $a, b, c, d$ are four integers such that $a+2 b+2 c+3 d=3 n$. Then, by $4.2,(5)$, and (6), with the same notation as in section 1.7 ,

$$
\begin{aligned}
N_{a, b, c, d} & =\int_{G(3, n+3)} \sigma_{1}^{a} \cdot \sigma_{2}^{b} \cdot \sigma_{(134)}^{c} \cdot \sigma_{(135)}^{d} \cap[G(3, n+3)] \\
& =\int_{n+3} D_{1}^{a} \cdot D_{2}^{b} \cdot\left(\Delta_{(134)}(D)\right)^{c} \cdot\left(\Delta_{(135)}(D)\right)^{d}\left(\epsilon^{1} \wedge \epsilon^{2} \wedge \epsilon^{3}\right)
\end{aligned}
$$

is the number of projectively non equivalent rational plane curves of degree $n+2$ having $a$ flexes, $b$ hyperflexes, $c$ cusps, and $d$ tacnodes at $a+b+c+d$ distinct points. By definition of $\Delta$ (section 1.6) and of $D_{t}^{-1}$ (section 1.8),

$$
\Delta_{(134)}(D)=D_{1}^{2}-D_{2}=\bar{D}_{2} \quad \text { and } \quad \Delta_{(135)}(D)=D_{1} D_{2}-D_{3}=D_{1} \bar{D}_{2}-\bar{D}_{3}
$$


In fact, $D_{t} \circ D_{t}^{-1}=1$ implies that $D_{3}=D_{2} \bar{D}_{1}-D_{1} \bar{D}_{2}+\bar{D}_{3}$ and since $\bar{D}_{1}=D_{1}$ one gets the last member of the second equality of (25). Thus,

$$
\begin{aligned}
N_{a, b, c, d} & =\int_{n+3} D_{1}^{a} \cdot D_{2}^{b} \cdot \bar{D}_{2}^{c} \cdot\left(D_{1} \bar{D}_{2}-\bar{D}_{3}\right)^{d}\left(\epsilon^{1} \wedge \epsilon^{2} \wedge \epsilon^{3}\right) \\
& =\sum_{d^{\prime}=0}^{d}(-1)^{d^{\prime}} \int_{n+3}\left(\begin{array}{c}
d \\
d^{\prime}
\end{array}\right) D_{1}^{a+d^{\prime}} D_{2}^{b} \bar{D}_{2}^{c+d^{\prime}} \bar{D}_{3}^{d-d^{\prime}}\left(\epsilon^{1} \wedge \epsilon^{2} \wedge \epsilon^{3}\right)
\end{aligned}
$$

Theorem 4.8. The number $N_{a, b, c, d}$ is given by

$$
N_{a, b, c, d}=\sum_{\substack{0 \leq d^{\prime} \leq d \\
\boldsymbol{\beta} \in p_{4}(b) \\
\gamma \in p_{3}\left(c+d^{\prime}\right)}}(-1)^{d^{\prime}}\left(\begin{array}{c}
d \\
d^{\prime}
\end{array}\right)\left(\begin{array}{c}
b \\
\boldsymbol{\beta}
\end{array}\right)\left(\begin{array}{c}
c+d^{\prime} \\
\gamma
\end{array}\right) \omega_{\left(i_{1}(d, \boldsymbol{\beta}, \boldsymbol{\gamma}), i_{2}(d, \boldsymbol{\beta}, \boldsymbol{\gamma}), i_{3}(d, \boldsymbol{\beta}, \boldsymbol{\gamma})\right)}
$$

where

$$
\left\{\begin{array}{l}
i_{1}(d, \boldsymbol{\beta}, \boldsymbol{\gamma})=1+d-d^{\prime}+\gamma_{2}+\gamma_{3}+\beta_{1} \\
i_{2}(d, \boldsymbol{\beta}, \boldsymbol{\gamma})=2+d-d^{\prime}+\gamma_{1}+\gamma_{3}+\beta_{2}+2 \beta_{4} \\
i_{3}(d, \boldsymbol{\beta}, \boldsymbol{\gamma})=3+d-d^{\prime}+\gamma_{1}+\gamma_{2}+2 \beta_{3}+\beta_{2}
\end{array}\right.
$$

Proof. By applying the formulas proven in section 3. First one computes

$$
\begin{aligned}
\bar{D}_{2}^{c+d^{\prime}} \bar{D}_{3}^{d-d^{\prime}} & \left(\epsilon^{1} \wedge \epsilon^{2} \wedge \epsilon^{3}\right) \\
& =\bar{D}_{2}^{c+d^{\prime}}\left(\epsilon^{1+d-d^{\prime}} \wedge \epsilon^{2+d-d^{\prime}} \wedge \epsilon^{3+d-d^{\prime}}\right) \\
& =\sum_{\gamma \in p_{3}\left(c+d^{\prime}\right)}\left(\begin{array}{c}
c+d^{\prime} \\
\gamma
\end{array}\right) \epsilon^{1+d-d^{\prime}+\gamma_{2}+\gamma_{3}} \wedge \epsilon^{2+d-d^{\prime}+\gamma_{1}+\gamma_{3}} \wedge \epsilon^{3+d-d^{\prime}+\gamma_{1}+\gamma_{2}},
\end{aligned}
$$

where the first equality is by (16) and the second one is by Proposition 3.2. Moreover

$$
\begin{aligned}
D_{1}^{a+d} D_{2}^{b}\left(\epsilon^{1+d-d^{\prime}+\gamma_{2}+\gamma_{3}} \wedge \epsilon^{2+d-d^{\prime}+\gamma_{1}+\gamma_{3}} \wedge \epsilon^{3+d-d^{\prime}+\gamma_{1}+\gamma_{2}}\right) & \\
& =\sum\left(\begin{array}{l}
b \\
\boldsymbol{\beta}
\end{array}\right) D_{1}^{a+d+\beta_{1}}\left(\epsilon^{i_{1}(d, \boldsymbol{\beta}, \gamma)} \wedge \epsilon^{i_{2}(d, \boldsymbol{\beta}, \gamma)} \wedge \epsilon^{i_{3}(d, \boldsymbol{\beta}, \gamma)}\right) \\
& =\sum\left(\begin{array}{l}
b \\
\boldsymbol{\beta}
\end{array}\right) \omega_{\left(i_{1}(d, \boldsymbol{\beta}, \gamma), i_{2}(d, \boldsymbol{\beta}, \boldsymbol{\gamma}), i_{3}(d, \boldsymbol{\beta}, \boldsymbol{\gamma})\right)}
\end{aligned}
$$

with $i_{1}(d, \boldsymbol{\beta}, \boldsymbol{\gamma}), i_{2}(d, \boldsymbol{\beta}, \boldsymbol{\gamma}), i_{3}(d, \boldsymbol{\beta}, \boldsymbol{\gamma})$ given by (26). Putting all together, one gets precisely the claimed formula for $N_{a, b, c, d}$

4.9 Counting webs on $\mathbb{P}^{1}$. Let $V$ be a base point free web on $\mathbb{P}^{1}$ and let $\phi: \mathbb{P}^{1} \rightarrow \mathbb{P}(V)$ be the induced morphism. Let $P \in \mathbb{P}^{1}$. We shall say that $Q:=\phi(P)$ is a stall, a hyperstall, a flex, a cusp, if the $V$-ramification sequence at $P$ is, respectively, 
$(1,2,3,5),(1,2,3,6),(1,2,4,5),(1,3,4,5)$. At a stall (resp. at a hyperstall) $Q \in C$ the osculating plane meets the curve at $Q$ with multiplicity 4 (resp. with multiplicity 5 ). If $Q$ is a flex, all the planes of the pencil containing the tangent line to $Q$ meets the curve with multiplicity at least 3 . If $Q$ is a cusp, the local analytic equation of $C$ at $Q$ is precisely $y^{2}-x^{3}=0$. Imitating what we did for rational plane curves, we may write a formula counting the number (with multiplicity) of all the (projectively non equivalent) rational space curves having $a$ stalls, $b$ hyperstalls, $c$ flexes, and $d$ cusps, at $a+b+c+d$ distinct points such that $a+2 b+2 c+3 d=4 n$. Such a number is counted by the integral (see $4.2,1.7,1.8$ )

$$
\begin{aligned}
f_{a, b, c, d} & :=\int_{G(4, n+4)} \sigma_{1}^{a} \sigma_{2}^{b} \sigma_{1245}^{c} \sigma_{1345}^{d} \cap[G(4, n+4)] \\
& =\int_{n+4} D_{1}^{a} D_{2}^{b} \bar{D}_{2}^{c} \bar{D}_{3}^{d}\left(\epsilon^{1} \wedge \epsilon^{2} \wedge \epsilon^{3} \wedge \epsilon^{4}\right),
\end{aligned}
$$

i.e., the coefficient of $\pi_{4, n+4}$ (see section 1.2), in the expansion of

$$
D_{1}^{a} D_{2}^{b} \bar{D}_{2}^{c} \bar{D}_{3}^{d}\left(\epsilon^{1} \wedge \epsilon^{2} \wedge \epsilon^{3} \wedge \epsilon^{4}\right)
$$

Theorem 4.10. The number $f_{a, b, c, d}$ is given by

$$
f_{a, b, c, d}=\sum_{\substack{\boldsymbol{\beta} \in p_{5}(b) \\
\boldsymbol{\gamma} \in p_{4}(c) \\
\boldsymbol{\delta} \in p_{4}(d) \\
0 \leq l \leq \beta_{2}+\beta_{3} \\
0 \leq m \leq \gamma_{1}+\gamma_{2}}}\left(\begin{array}{l}
b \\
\boldsymbol{\beta}
\end{array}\right)\left(\begin{array}{l}
c \\
\boldsymbol{\gamma}
\end{array}\right)\left(\begin{array}{l}
d \\
\boldsymbol{\delta}
\end{array}\right)\left(\begin{array}{c}
\beta_{2}+\beta_{3} \\
l
\end{array}\right)\left(\begin{array}{c}
\gamma_{1}+\gamma_{2} \\
m
\end{array}\right) \omega_{I(\boldsymbol{\beta}, \boldsymbol{\gamma}, \boldsymbol{\delta} ; l, m)}
$$

having set

$$
\begin{aligned}
& I(\boldsymbol{\beta}, \boldsymbol{\gamma}, \boldsymbol{\delta} ; l, m) \\
& \quad=\left(i_{1}(\boldsymbol{\beta}, \boldsymbol{\gamma}, \boldsymbol{\delta} ; l, m), i_{2}(\boldsymbol{\beta}, \boldsymbol{\gamma}, \boldsymbol{\delta} ; l, m), i_{3}(\boldsymbol{\beta}, \boldsymbol{\gamma}, \boldsymbol{\delta} ; l, m), i_{4}(\boldsymbol{\beta}, \boldsymbol{\gamma}, \boldsymbol{\delta} ; l, m)\right)
\end{aligned}
$$

with

$$
\left\{\begin{array}{l}
i_{1}(\boldsymbol{\beta}, \boldsymbol{\gamma}, \boldsymbol{\delta} ; l, m)=1+\sum_{j \neq 1} \delta_{j}+\gamma_{1}+\gamma_{4}+\beta_{1}, \\
i_{2}(\boldsymbol{\beta}, \boldsymbol{\gamma}, \boldsymbol{\delta} ; l, m)=2+\sum_{j \neq 2} \delta_{j}+\gamma_{2}+\gamma_{4}+\beta_{2}+2 \beta_{5}, \\
i_{3}(\boldsymbol{\beta}, \boldsymbol{\gamma}, \boldsymbol{\delta} ; l, m)=3+\sum_{j \neq 3} \delta_{j}+\gamma_{3}+\beta_{3}+l+m, \\
i_{4}(\boldsymbol{\beta}, \boldsymbol{\gamma}, \boldsymbol{\delta} ; l, m)=4+\sum_{j \neq 4} \delta_{j}+\gamma_{1}+\gamma_{2}+\gamma_{3}+\beta_{2}+2 \beta_{4}+\beta_{3}-l-m
\end{array}\right.
$$

(where $\omega_{(\boldsymbol{\beta}, \boldsymbol{\gamma}, \boldsymbol{\delta} ; l, m)}=0$ if $i_{p}(\boldsymbol{\beta}, \boldsymbol{\gamma}, \boldsymbol{\delta} ; l, m)>n+4$ for some $\left.p \in\{1,2,3,4\}\right)$. 
Proof. The proof is straightforward. By formula (17), one first has

$$
\begin{aligned}
D_{1}^{a} D_{2}^{b} \bar{D}_{2}^{c} \bar{D}_{3}^{d}\left(\epsilon^{1} \wedge \epsilon^{2} \wedge \epsilon^{3} \wedge \epsilon^{4}\right) \\
=D_{1}^{a} D_{2}^{b} \bar{D}_{2}^{c} \sum_{\delta \in p_{4}(d)} \frac{d !}{\delta !} \epsilon^{1+\sum_{j \neq 1} \delta_{j}} \wedge \epsilon^{2+\sum_{j \neq 2} \delta_{j}} \wedge \epsilon^{3+\sum_{j \neq 3} \delta_{j}} \wedge \epsilon^{4+\sum_{j \neq 4} \delta_{j}} .
\end{aligned}
$$

One now applies (iii) of 3.3 , getting

$$
D_{1}^{a} D_{2}^{b} \sum_{\substack{\boldsymbol{\delta} \in p_{4}(d) \\
\boldsymbol{\gamma} \in p_{4}(c) \\
0 \leq m \leq \gamma_{1}+\gamma_{2}}}\left(\begin{array}{l}
c \\
\gamma
\end{array}\right)\left(\begin{array}{l}
d \\
\boldsymbol{\delta}
\end{array}\right)\left(\begin{array}{c}
\gamma_{1}+\gamma_{2} \\
m
\end{array}\right) \epsilon^{i_{1}(\boldsymbol{\gamma}, \boldsymbol{\delta} ; m)} \wedge \epsilon^{i_{2}(\boldsymbol{\gamma}, \boldsymbol{\delta} ; m)} \wedge \epsilon^{i_{3}(\boldsymbol{\gamma}, \delta ; m)} \wedge \epsilon^{i_{4}(\boldsymbol{\gamma}, \boldsymbol{\delta} ; m)},
$$

where

$$
\left\{\begin{array}{l}
i_{1}(\boldsymbol{\gamma}, \boldsymbol{\delta} ; m)=1+\sum_{j \neq 1} \delta_{j}+\gamma_{1}+\gamma_{4}, \\
i_{2}(\boldsymbol{\gamma}, \boldsymbol{\delta} ; m)=2+\sum_{j \neq 2} \delta_{j}+\gamma_{2}+\gamma_{4}, \\
i_{3}(\boldsymbol{\gamma}, \boldsymbol{\delta} ; m)=3+\sum_{j \neq 3} \delta_{j}+\gamma_{3}+m, \\
i_{4}(\boldsymbol{\gamma}, \boldsymbol{\delta} ; m)=4+\sum_{j \neq 4} \delta_{j}+\gamma_{1}+\gamma_{2}+\gamma_{3}-m .
\end{array}\right.
$$

Finally, applying formula (ii) of 3.3 , this is equal to

$$
\sum_{\substack{\boldsymbol{\beta} \in p_{5}(b) \\
\boldsymbol{\gamma} \in p_{4}(c) \\
\boldsymbol{\delta} \in p_{4}(d) \\
0 \leq l \leq \beta_{2}+\beta_{3} \\
0 \leq m \leq \gamma_{1}+\gamma_{2}}}\left(\begin{array}{l}
b \\
\boldsymbol{\beta}
\end{array}\right)\left(\begin{array}{l}
c \\
\gamma
\end{array}\right)\left(\begin{array}{l}
d \\
\boldsymbol{\delta}
\end{array}\right)\left(\begin{array}{c}
\beta_{2}+\beta_{3} \\
l
\end{array}\right)\left(\begin{array}{c}
\gamma_{1}+\gamma_{2} \\
m
\end{array}\right) D_{1}^{a+\beta_{1}} \boldsymbol{\epsilon}^{I(\beta, \gamma, \delta ; l, m)},
$$

where $I(\boldsymbol{\beta}, \boldsymbol{\gamma}, \boldsymbol{\delta} ; l, m)$ is given precisely by (28). Taking integrals, one gets precisely formula (27).

4.11. Let $a=c=d=0$. Then $b=2 n$. For each $\boldsymbol{\beta} \in p_{5}(2 n)$, let

$$
I(\boldsymbol{\beta}, l)=\left(1+\beta_{1}, 2+\beta_{2}+2 \beta_{5}, 3+\beta_{3}+l, 4+\beta_{2}+\beta_{3}+2 \beta_{4}-l\right) .
$$

Then

$$
\mathrm{HS}_{n}:=f_{0,2 n, 0,0}=\sum_{\substack{\boldsymbol{\beta} \in p_{5}(2 n) \\
0 \leq l \leq \boldsymbol{\beta}_{2}+\beta_{3}}}\left(\begin{array}{c}
2 n \\
\boldsymbol{\beta}
\end{array}\right)\left(\begin{array}{c}
\beta_{2}+\beta_{3} \\
l
\end{array}\right) \omega_{I(\boldsymbol{\beta} ; l)},
$$

is the number (with multiplicities) of rational space curves having $2 n$ hyperstalls at $2 n$ prescribed distinct points. 
Similarly, if $a, b, d=0$, then $c=2 n$. For each $\gamma \in p_{4}(2 n)$, let

$$
I(\gamma ; m)=\left(1+\gamma_{1}+\gamma_{4}, 2+\gamma_{2}+\gamma_{4}, 3+\gamma_{3}+m, 4+\gamma_{1}+\gamma_{2}+\gamma_{3}-m\right) .
$$

Thus,

$$
f_{0,0,2 n, 0}=\sum_{\substack{\gamma \in p_{4}(2 n) \\
0 \leq m \leq \gamma_{1}+\gamma_{2}}}\left(\begin{array}{c}
2 n \\
\gamma
\end{array}\right)\left(\begin{array}{c}
\gamma_{1}+\gamma_{2} \\
m
\end{array}\right) \omega_{I(\boldsymbol{\gamma} ; m)},
$$

which is our way to answer to Ranestad's original question.

Acknowledgements. Our first debt of gratitude is with K. Ranestad who asked us the main question giving rise to this paper, and with D. Laksov for discussions and constant support. For technical assistance we thank G. Ghibò, S. Berrone, A. Bigatti, but especially J. M. Økland who taught us the use of Macaulay2 and improved our first primitive CoCoA code. We also thank Paolo Aluffi, for useful comments. Finally, we are extremely grateful to the referee who, with his/her remarks and suggestions, is responsible for the many substantial improvements with respect to a previous version of this paper.

\section{References}

[1] P. J. Cameron, Combinatorics: Topics, techniques, algorithms, Cambridge University Press, Cambridge, 1994.

[2] Cocoa team, CoCoA: A system for doing computations in Commutative Algebra, available at http://cocoa.dima. unige.it.

[3] J. Cordovez, L. Gatto, and T. Santiago, CoCoA code for the number of rational space curves with the maximum number of hyperstalls, available at http://calvino.polito.it/ gatto/public/ English/research/SCGA/cgscodes.htm.

[4] D. Eisenbud and J. Harris, Divisors on general curves and cuspidal rational curves, Invent. Math. 74 (1983), no. 3, 371-418.

[5] W. Fulton, Intersection theory, Ergebnisse der Mathematik und ihrer Grenzgebiete (3), vol. 2, Springer-Verlag, Berlin, 1984.

[6] L. Gatto, Schubert calculus via Hasse-Schmidt derivations, Asian J. Math. 9 (2005), no. 3, $315-321$.

[7] _ Schubert calculus: An algebraic introduction, Publicações Matemáticas do IMPA, Instituto Nacional de Matemática Pura e Aplicada (IMPA), Rio de Janeiro, 2005.

[8] L. Gatto and T. Santiago, Schubert Calculus on Grassmann Algebra, Canad. Math. Bulletin, to appear, available at arXiv:math.AG/0702759v1.

[9] D. R. Grayson and M. E. Stillmand, Schubert2, computations of characteristic classes for varieties without equations, part of Macaulay 2, a software system for research in algebraic geometry, available at http://www.math.uiuc.edu/Macaulay2.

[10] Ph. Griffiths and J. Harris, Principles of algebraic geometry, Pure and Applied Mathematics, Wiley-Interscience, New York, 1978. 
[11] S. Katz and S. A. Strømme, "Schubert", a Maple $\left.{ }^{(}\right)$package for intersection theory and enumerative geometry, available at http://math.uib.no/schubert/.

[12] V. Kharlamov and F. Sottile, Maximally inflected real rational curves, Mosc. Math. J. 3 (2003), no. 3, 947-987, 1199-1200.

[13] D. Laksov and A. Thorup, A determinantal formula for the exterior powers of the polynomial ring, Indiana Univ. Math. J. 56 (2007), no. 2, 825-845.

[14] _ Schubert Calculus on Grassmannians and exterior products, Indiana Univ. Math. J., to appear.

[15] J. M. Økland and I. Vainsencher, 2007, private comunication.

[16] B. Osserman, The number of linear series on curves with given ramification, Int. Math. Res. Not. 47 (2003), 2513-2527.

[17] T. Santiago, Schubert calculus on a Grassmann algebra, Ph. D. Thesis, Politecnico di Torino, 2006.

[18] I. Scherbak, Gaudin's model and the generating function of the Wronski map, Geometry and Topology of Caustics (Warsaw, 2002), Banach Center Publ., vol. 62, Polish Acad. Sci., Warsaw, 2004, pp. 249-262.

[19] I. Scherbak and A. Varchenko, Critical points of functions, $\mathfrak{s l}_{2}$ representations, and Fuchsian differential equations with only univalued solutions, Mosc. Math. J. 3 (2003), no. 2, 621-645.

[20] H. Schubert, Anzahl-Bestimmungen für lineare Räume: Beliebiger Dimension, Acta Math. 8 (1886), no. 1, 97-118. 